\title{
Endpoint relations for baryons
}

\author{
Gudrun Hiller $^{a}$ and Roman Zwicky ${ }^{b}$ \\ ${ }^{a}$ Institut für Physik, Technische Universität Dortmund, \\ D-44221 Dortmund, Germany \\ ${ }^{b}$ Higgs Centre for Theoretical Physics, School of Physics and Astronomy, University of Edinburgh, \\ Edinburgh EH9 3JZ, Scotland \\ E-mail: gudrun.hiller@uni-dortmund.de, roman.zwicky@ed.ac.uk
}

ABSTRACT: Following our earlier work we establish kinematic endpoint relations for baryon decays using the Wigner-Eckart theorem and apply them to $\frac{1}{2} \rightarrow \frac{1}{2}$ and $\frac{1}{2} \rightarrow \frac{3}{2}$ baryon transitions. We provide angular distributions at the kinematic endpoint which hold for the generic $d=6$ model-independent effective Hamiltonian and comment on the behaviour in the vicinity of the endpoint. Moreover, we verify the endpoint relations, using an explicit form factor parametrisation, and clarify constraints on helicity-based form factors to evidence endpoint relations. Our results provide guidance for phenomenological parameterisations, consistency checks for theory computations and experiment. Results are applicable to ongoing and future new physics searches at LHCb, BES III and Belle II with rare semileptonic-, dineutrino-and charged-modes, which include $\Lambda_{b} \rightarrow \Lambda^{(*)} \ell \ell, \Lambda_{b} \rightarrow \Lambda^{(*)} \nu \nu$, $\Omega_{b} \rightarrow \Omega \ell \ell, \Lambda_{c} \rightarrow p \ell \ell, \Sigma \rightarrow p \ell \ell$ and $\Lambda_{b} \rightarrow \Lambda_{c}^{(*)} \ell \nu$.

KeYwORDS: Heavy Quark Physics, CP violation

ARXiv EPRINT: 2107.12993 


\section{Contents}

1 Introduction 1

2 The main ideas behind endpoint relations and symmetries $\quad 2$

2.1 Helicity formalism for $d=6$ effective Hamiltonian 2

2.2 Three pathways to endpoint relations 3

3 The baryon decays $\frac{1}{2}^{+} \rightarrow \frac{1}{2}\left(\frac{3}{2}\right)^{ \pm} \ell_{1} \bar{\ell}_{2}$

3.1 The baryonic form factors 5

3.1.1 The $\Lambda_{J^{P}=\frac{1}{2}}{ }^{+} \rightarrow \Lambda_{J^{P}=\frac{1}{2}^{ \pm}}^{\prime}$ form factors $\quad 5$

3.1.2 The $\Lambda_{J^{P}=\frac{1}{2}}{ }^{+} \rightarrow \Lambda_{J^{P}=\frac{3}{2}}^{*}$ form factors 6

3.2 The baryonic helicity amplitudes and verification of endpoint relations $\quad 7$

3.2.1 The $\Lambda_{J^{P}=\frac{1}{2}}{ }^{+} \rightarrow \Lambda_{J^{P}=\frac{1^{ \pm}}{}}^{\prime}$ helicity amplitudes $\quad 7$

3.2.2 The $\Lambda_{J^{P}=\frac{1}{2}}{ }^{+} \rightarrow \Lambda_{J^{P}=\frac{3}{2}}^{*}$ helicity amplitudes 8

3.3 Specific endpoint features compared with $B \rightarrow V \ell_{1} \bar{\ell}_{2}$

4 Constraints on helicity based form factors $\quad 10$

$\begin{array}{ll}4.1 \text { Comparison with the literature } & 12\end{array}$

5 Angular distributions $\quad \mathbf{1 2}$

5.1 The $\Lambda_{J^{P}=\frac{1}{2}}{ }^{+} \rightarrow \Lambda_{J^{P}=\frac{1}{2}}^{\prime}{ }^{ \pm}$angular distribution at the endpoint 13

5.2 The $\Lambda_{J^{P}=\frac{1}{2}}{ }^{+} \rightarrow \Lambda_{J^{P}=\frac{3}{2}}^{\prime}{ }^{ \pm}$angular distribution at the endpoint 14

$\begin{array}{lll}5.3 & \text { In the vicinity of the endpoint } & 15\end{array}$

$6 \quad$ Summary and conclusions $\quad 16$

$\begin{array}{ll}\text { A Conventions } & 17\end{array}$

$\begin{array}{ll}\text { A.1 Polarisation vectors } & 17\end{array}$

$\begin{array}{lll}\text { A.2 Dirac algebra } & 18\end{array}$

$\begin{array}{lll}\text { A.3 The } \frac{3}{2} \text {-spinor } & 18\end{array}$

B The tensor form factor relation due to $\sigma^{\alpha \beta} \gamma_{5}=-\frac{i}{2} \varepsilon^{\alpha \beta \gamma \delta} \sigma_{\gamma \delta} \quad 19$

$\begin{array}{ll}\text { B.1 } \Lambda_{J^{P}=\frac{1}{2}}{ }^{+} \rightarrow \Lambda_{J^{P}=\frac{1}{2}}^{\prime} \pm^{\text {-case }} & 19\end{array}$

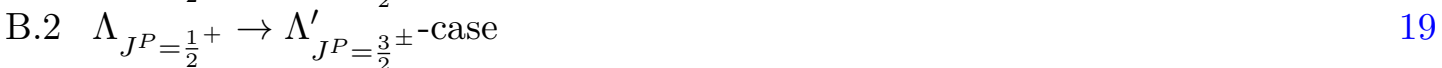




\section{Introduction}

The kinematic endpoint of a decay is characterised by the velocities, of some of its decaying particles, approaching zero. This results in the restoration of spherical symmetry and leads to enhanced symmetries in the helicity amplitudes [1], which crucially extends to effective field theories such as $c \rightarrow u \ell^{+} \ell^{-}$or $b \rightarrow s \ell^{+} \ell^{-}[2] .^{1}$ The ideas behind this symmetry are completely general and apply at the non-perturbative level and any decay types including the baryonic ones which are the focus of this presentation. At the level of the differential decay rate the relations result in exact predictions at the kinematic endpoint [2]. We refer to these predictions as "endpoint relations". On a pragmatic level they provide guiding for fit-parameterisation, non-trivial consistency checks for theoretical computations and experiment itself.

The specific presentation of this paper applies to the baryonic decays ${ }^{2}$

$$
\begin{array}{ll}
\Lambda_{\frac{1}{2}}+\rightarrow \Lambda_{\frac{1}{2}}^{\prime} \ell_{1} \bar{\ell}_{2}, & \text { e.g. } \Lambda_{c} \rightarrow p \ell^{+} \ell^{-}, \\
\Lambda_{\frac{1}{2}}{ }^{+} \rightarrow \Lambda_{\frac{3}{2}}^{*} \ell_{1} \bar{\ell}_{2} & \text { e.g. } \Omega_{c}^{0} \rightarrow \Omega^{-} \ell^{+} \nu,
\end{array}
$$

described by a $d=6$ effective Hamiltonian. ${ }^{3}$ This includes decays of the semileptonic charged and flavour changing neutral current (FCNC) type into charged leptons and neutrinos. Such modes have been known for a long time as sensitive probes of flavour physics in and beyond the Standard Model. A vast number of theoretical and experimental studies for $K, D, B$-meson decays exists $[4,5]$.

Rare decays of beauty, charm and strange baryons, such as $\Lambda_{b} \rightarrow \Lambda \ell \ell$ decays offer new ways to test the SM and look for new physics, complementing searches with mesons. Due to different theoretical and experimental systematics, caused by the interplay of strong \& weak dynamics and production \& reconstruction efficiencies respectively. In addition, baryon modes provide opportunities on their own, including polarisation studies based on polarized baryon production or angular distributions of the decay products of the final baryon.

Recently, observables induced by FCNC $b \rightarrow s \ell^{+} \ell^{-}$transitions have been reported that hint at new physics and specifically new phenomenon, violating lepton flavour universality $[6,7]$. This includes studies with $\Lambda_{b}$-decays [8], consistent with the flavour anomalies evidenced in $B$-decays [9], although with currently larger uncertainties. Cross-checking findings from $B \rightarrow K^{(*)} \mu^{+} \mu^{-}$decays with other modes induced by the same FCNC transition provides a natural and important next step to understand the origin of the flavour anomalies. It is therefore timely to put baryonic modes at par with the mesonic ones. For baryonic modes there are a number of form factor computations, e.g. lattice [10-14] and sum rules [15, 16], but no long-distance computations in QCD-factorisation or light-cone sum rules.

\footnotetext{
${ }^{1}$ Alternatively, it can be viewed as the partial wave expansion applied to effective field theories.

${ }^{2}$ The baryons subscripts denote the $J^{P}$ quantum numbers and the adaption to the case where the initial state baryon is $J^{P}=\frac{1}{2}^{-}$is straightforward as one can simply perform a parity transformation.

${ }^{3}$ There are two loopholes. Firstly, QED-corrections break the factorisation into a hadronic and leptonic helicity amplitude (cf. section 5 in [3] for a discussion thereof and brief comments in section 5.3). Second, new light-physics beyond the Standard Model for which the effective theory approach becomes suboptimal. Whereas the first are presumably not too large the latter are an exciting possibility for which endpoint relations are yet another tool to search for them.
} 
Rare baryonic decays are suitable for experimental study at high luminosity flavour facilities, such as LHCb [17], Belle II [18], BES III [19], and possible future machines [20]. Efforts exist for Hyperons [21] and in charm [22-25]. In beauty, the situation is more advanced, and first studies with angular distributions for $b$-baryon decays are available [26], as well as prospects for decays to spin $3 / 2$ baryons, such as $\Lambda_{b} \rightarrow \Lambda(1520) \ell \ell$ [27]. The $\Lambda(1520)$ has received attention as it is the most pronounced resonance decaying strongly to $p K$ [28].

The paper is organised as follows. In section 2 we review the ideas behind the endpoint relations for helicity amplitudes from various viewpoints. In section 3 the concepts are applied to the decays in (1.1) at the level of the form factors. Moreover constraints on helicity-based form factors are discussed in section 4 which are needed to evidence form factors relation in this parametrisation. In section 5 we apply the endpoint relation to angular distribution and derive exact relations valid at the endpoint. The paper ends with summary and conclusions in section 6. Appendices A and B contain conventions and a form factor derivation respectively.

\section{The main ideas behind endpoint relations and symmetries}

We would like to present three ideas to understand the endpoint relations in increasing degree of generality and rigour: rotational symmetry, reduction of invariants and the Wigner-Eckart theorem. Before doing this we briefly summarise, for the reader unfamiliar with the subject, the helicity formalism.

\subsection{Helicity formalism for $d=6$ effective Hamiltonian}

The description of $P \rightarrow V\left(\rightarrow P_{1} P_{2}\right) V^{\prime}$ decays in the helicity formalism goes back to the seminal work of Jacob and Wick [29] reworked in excellent lecture notes [30]. The extension to $P \rightarrow V \ell_{1} \bar{\ell}_{2}$ or $\Lambda \rightarrow \Lambda^{\prime} \ell_{1} \bar{\ell}_{2}$, involving an auxiliary timelike polarisation, has been known for some time e.g. [31] and [32]. The extension beyond the dimension six Hamiltonian has been worked out in full generality in [3]. Here we focus on the helicity formalism as applied to the dimension six effective Hamiltonian which contains operators of the type $H_{\mathrm{eff}}^{d=6} \supset \bar{u} \gamma_{\alpha} c \bar{\ell}_{1} \gamma^{\alpha} \ell_{2}$. In what follows it is sufficient to introduce the following six operators for the hadronic part

$$
O_{S(P)} \equiv \bar{u}\left(\gamma_{5}\right) c, \quad O_{V(A)}^{\mu} \equiv \bar{u} \gamma^{\mu}\left(\gamma_{5}\right) c, \quad O_{T_{(5)}}^{\mu \nu} \equiv \bar{u} \sigma^{\mu \nu}\left(\gamma_{5}\right) c
$$

and analogous ones for the leptons. In the absence of QED-corrections, the matrix element factorises into a hadronic and a leptonic part

$$
\begin{aligned}
\left\langle p \ell_{1} \overline{\ell_{2}}\left|H_{\mathrm{eff}}^{c \rightarrow u \ell^{+} \ell^{-}}\right| \Lambda_{c}\right\rangle= & \sum_{i=S, P}\left\langle p\left|H_{i}\right| \Lambda_{c}\right\rangle\left\langle\ell_{1} \overline{\ell_{2}}\left|L_{i}\right| 0\right\rangle+ \\
& \sum_{i=V, A}\left\langle p\left|H_{i}^{\mu}\right| \Lambda_{c}\right\rangle\left\langle\ell_{1} \overline{\ell_{2}}\left|L_{i}^{\mu^{\prime}}\right| 0\right\rangle g_{\mu \mu^{\prime}}+ \\
& \sum_{i=T, T_{5}}\left\langle p\left|H_{i}^{\mu \nu}\right| \Lambda_{c}\right\rangle\left\langle\ell_{1} \overline{\ell_{2}}\left|L_{i}^{\mu^{\prime} \nu^{\prime}}\right| 0\right\rangle g_{\mu \mu^{\prime}} g_{\nu \nu^{\prime}},
\end{aligned}
$$


where the hadronic current is $H_{i}^{\mu \ldots} \propto O_{i}^{\mu \ldots}$ in (2.1) and the leptonic currents are given by $L_{S}=\bar{\ell}_{1} \ell_{2}$ etc. The discussion in oversimplified in that we do not discuss different chiralities but there is no loss in doing so.

The key insight in applying the helicity formalism to effective field theories comes from the observation that the Minkowski metric can be replaced by the sum of the usual helicity vectors plus an auxiliary timelike polarisation $\beta(q, t)=q / \sqrt{q^{2}}$, transverse to the physical polarisation vectors (for which $q \cdot \beta(q, \pm, 0)=0$ ). The completeness relation then reads

$$
\sum_{\lambda, \lambda^{\prime} \in\{t, \pm, 0\}} \beta^{\mu}(q, \lambda) \beta^{* \nu}\left(q, \lambda^{\prime}\right) G_{\lambda \lambda^{\prime}}=g^{\mu \nu}, \quad G_{\lambda \lambda^{\prime}}=\operatorname{diag}(1,-1,-1,-1),
$$

where the first entry in $G_{\lambda \lambda^{\prime}}$ refers to $\lambda=\lambda^{\prime}=t$ and a complete set of helicity vectors is given in appendix A.1. In this way Minkowski indices are traded for helicity indices. This then defines the helicity amplitude (HA) characterised by its helicities. Note that in the decay one helicity can be eliminated by generalised helicity conservation. In this counting the timelike helicity counts as zero since it does not transform under a rotation in the plane transverse to the movement $[2,3]$. We choose the spin quantisation axis to be the $q$-direction in the $\Lambda_{c}$ restframe. Parameterising the HA as

$$
H_{\lambda_{q} \bar{\sigma}}^{V} \equiv\left\langle p(\sigma)\left|H_{\mu}^{V}\right| \Lambda_{c}\left(\lambda_{c}\right)\right\rangle \beta^{* \mu}\left(q, \lambda_{q}\right)
$$

the helicity conservation equation reads

$$
\lambda_{c}=\lambda_{q}+\bar{\sigma}
$$

where the notation $\bar{\sigma} \equiv-\sigma$ is employed throughout and $\lambda_{q}$ is the helicity of the fictitious particle in the decay chain $\Lambda_{c} \rightarrow p+q\left(\rightarrow \ell_{1} \bar{\ell}_{2}\right)$. The lepton helicity amplitudes follow in the same way $\mathcal{L}_{\lambda_{1} \lambda_{2}}^{V} \equiv\left\langle\ell_{1} \bar{\ell}_{2}\left|L_{\mu}^{V}\right| 0\right\rangle \beta^{\mu}\left(q, \lambda_{q}\right)$ but are not the focus of this paper and their explicit expression can be found in appendix A.3 in [3].

\subsection{Three pathways to endpoint relations}

In explaining endpoint relations in terms of rotational symmetry, invariants and the WignerEckart theorem it is useful to write an explicit parametrisation of the momenta involved

$$
p^{\mu}=\left(m_{\Lambda_{c}}, 0,0,0\right), \quad p_{p(N)}^{\mu}=\left(E_{p(N)}, 0,0,-\kappa\right), \quad q^{\mu}=\left(q_{0}, 0,0, \kappa\right),
$$

where $p \equiv p_{p(N)}+q, q_{0}=\sqrt{q^{2}+\kappa^{2}}, E_{p(N)}=\sqrt{m_{p(N)}^{2}+\kappa^{2}}$ and

$$
\kappa \equiv \frac{\lambda^{1 / 2}\left(m_{\Lambda_{c}}^{2}, m_{p(N)}^{2}, q^{2}\right)}{2 m_{\Lambda_{c}}},
$$

is the velocity in the $\Lambda_{c}$ restframe (with $\lambda(x, y, z)=x^{2}+y^{2}+z^{2}-2 x z-2 x z-2 y z$ the Källén-function). The $\kappa \rightarrow 0$ limit makes the symmetry enhancement explicit: all 4-momenta are proportional to each other. The insightful viewpoint is that it is the external momenta $\kappa \neq 0$ that breaks the rotational symmetry. Upon discussing the rotationaland invariant-viewpoints it is convenient to consider $B(p) \rightarrow K^{*}\left(p_{p}\right)\left(\gamma^{*}(q) \rightarrow \ell^{+} \ell^{-}\right)$as this avoids dealing with Dirac spinors [2]. This is not an issue for the Wigner-Eckart viewpoint [1] which can be considered as one of its strengths. 
- Rotational symmetry. In the case of $B \rightarrow K^{*} \ell^{+} \ell^{-}, \lambda_{c}=0$ and $\sigma=\lambda_{q}$ by (2.5). Hence the entire HA is characterised by the helicity of the $K^{*}$ and one may write $H_{\lambda_{K^{*}}}^{V}$. At the kinematic endpoint rotational symmetry is restored and no direction is distinct from the viewpoint of the $K^{*}$-meson. Hence the HAs must be degenerate as found in the explicit evaluation at the level of the form factors (FFs) $H_{0}^{i}=-H_{+}^{i}=-H_{-}^{i}$ where $i=V, A{ }^{4}$ The respective signs depend on the conventions of the phases to which we will return to shortly.

- Reduction of invariants. A common way to count structures is to write down the number of invariants that can be formed. At the kinematic endpoint there is only a single non-vanishing invariant, namely $\eta^{*}\left(p_{p}, \lambda_{c}\right) \cdot \beta^{*}\left(q, \lambda_{q}\right)$. The momenta cannot be involved in scalar products with the polarisation vectors since they are vanishing by the transversity. The Levi-Civita tensor also vanishes as there are only three independent vectors. Hence there is only one structure contributing and the HAs are therefore degenerate.

- Wigner-Eckart theorem. The most powerful method is the application of the WignerEckart theorem due to (restored) rotational symmetry. The helicity information is then solely governed by the Clebsch-Gordan coefficients (CGC) and the reduced matrix element contains the only dynamic information [1]. Explicitly this statement reads for the case as hand (cf. (2.4))

$$
H_{\lambda_{q} \bar{\sigma}}^{i}=C_{\lambda_{c} \lambda_{q} \bar{\sigma}}^{\frac{1}{2} 1 j_{p}} M_{\frac{1}{2} 1 j_{\Lambda^{\prime}}}^{i}, \quad i=V, A, T, T_{5},
$$

where $C_{M m_{1} m_{2}}^{J j_{1} j_{2}}$ are the CGC which can be looked up in the particle data group resource [33] and $M$ is the reduced matrix element, independent of the helicity quantum numbers. It seems worthwhile to point out that whereas (2.8) always holds it does, as we shall see explicitly, hold trivially in half of the cases $(M=0)$ by parity conservation of QCD. Note that there is a unique invariant at the endpoint since $\mathrm{SU}(2)$ is a simply reducible group [1]. This means that in the direct product of irreducible representation of $\mathrm{SU}(2)$ the multiplicity of the Clebsch-Gordan series is either zero or one. It this was not the case then there could be several invariants with unknown relative coefficients between them and the distribution at the endpoint would not be completely independent of the dynamics of QCD.

Equipped with (2.8) we can then quote our main results

$$
\frac{1}{2}^{+} \rightarrow \frac{1}{2}^{ \pm}: \quad H_{0 \frac{1}{2}}^{i}: H_{1 \frac{1}{2}}^{i}=C_{\frac{1}{2} 0 \frac{1}{2}}^{\frac{1}{2} 1 \frac{1}{2}}: C_{\frac{1}{2} 1 \frac{1}{2}}^{\frac{1}{2} 1 \frac{1}{2}}=1:-\sqrt{2},
$$

and by parity the non-vanishing HAs are $i=A, T_{5}(V, T)$ (cf. section 3.1 just below for clarifications of the notation) and

$$
\frac{1}{2}^{+} \rightarrow \frac{3}{2}^{ \pm}: H_{0 \frac{1}{2}}^{i}: H_{1 \frac{1}{2}}^{i}: H_{\overline{1} \frac{3}{2}}^{i}=C_{\frac{1}{2} 0 \frac{1}{2}}^{\frac{1}{2} 1 \frac{3}{2}}: C_{\frac{1}{2} 1 \frac{1}{2}}^{\frac{1}{2} 1 \frac{3}{2}}: C_{\frac{1}{2} \overline{1} \frac{3}{2}}^{\frac{1}{2} 1 \frac{3}{2}}=1:-\sqrt{\frac{1}{2}}:-\sqrt{\frac{3}{2}},
$$

\footnotetext{
${ }^{4}$ For the tensor the same helicity structure emerges (cf. section B in [2]) $H_{t 0}^{T}=-H_{t+}^{T}=-H_{t-}^{T}$ and $H_{+-}^{T}=-H_{+0}^{T}=+H_{-0}^{T}$, where we correct a sign error in eq. (13) in [2], and remind the reader that $t$ counts as zero helicity in the conservation equation.
} 
$i=A, T_{5}(V, T)$ for the spin- $\frac{3}{2}$ baryon. In the remaining part of this paper we will verify these relations at the level of the short distance form factors and discuss the implications of the relations for the differential decay rates. The HAs for $\frac{1}{2}^{+} \rightarrow \frac{5}{2}^{ \pm}$transitions, which are of phenomenological interest, vanish at the endpoint by angular momentum conservation. They would not vanish if the transition operators were of spin 2 (cf. section 5.2 in [3]).

\section{The baryon decays $\frac{1}{2}^{+} \rightarrow \frac{1}{2}\left(\frac{3}{2}\right)^{ \pm} \ell_{1} \overline{\ell_{2}}$}

We choose to illustrate the endpoint relations in the baryonic decays by means of explicit form factors. We stress, once more, that the endpoint relations go beyond and also apply to any long distance matrix elements such as for example quark-loops originating from 4-quark operators of the $\bar{u} c \bar{q} q$-type.

\subsection{The baryonic form factors}

As for baryon form factors there does not seem to be a standard form factor basis unlike in the $B \rightarrow V$ case where the basis in $[34,35]$ is used in most analysis. The baryon form factors can be divided into helicity and non-helicity-based ones. We will choose to introduce a basis which is not helicity-based as this makes the analytic structure clearer and endpoint relations are satisfied automatically without the need to impose further constraints $\mathrm{cf}$. section 4. We do follow the logic of the basis in [34] in that the scalar form factor is separated and the constraint from the Ward identity or equation of motion is implemented by a relation to a single axial/vector form factor. Using our code we have checked that we agree with [36] in all HAs and with the vector HAs in [37] using their definitions.

\subsubsection{The $\Lambda_{J^{P}=\frac{1}{2}}{ }^{+} \rightarrow \Lambda_{J^{P}=\frac{1}{2}}^{\prime}{ }^{ \pm}$form factors}

For the $p\left(\frac{1}{2}^{+}\right)$final state, we define the dimensionless form factors by

$$
\begin{aligned}
\left\langle p\left(p_{p}, \sigma\right)\left|O_{V(A)}^{\mu}\right| \Lambda_{c}\left(p, \lambda_{c}\right)\right\rangle & =\bar{u}\left(\gamma_{5}\right)\left[P_{1}^{\mu} f_{1}^{V(A)}\left(q^{2}\right)+P_{2}^{\mu} f_{2}^{V(A)}\left(q^{2}\right)+P_{0}^{\mu} f_{0}^{V(A)}\left(q^{2}\right)\right] u \\
\left\langle p\left(p_{p}, \sigma\right)\left|O_{T_{(5)}}^{\mu}\right| \Lambda_{c}\left(p, \lambda_{c}\right)\right\rangle & =\bar{u}\left(\gamma_{5}\right)\left[P_{1}^{\mu} t_{1}^{V(A)}\left(q^{2}\right)+P_{2}^{\mu} t_{2}^{V(A)}\left(q^{2}\right)\right] u
\end{aligned}
$$

which is an alternative to $(3.1)$

$$
P_{0}^{\mu}=\frac{\hat{q}^{\mu}}{\hat{q}^{2}} \not{q}, \quad P_{1}^{\mu}=\hat{q}^{2} \gamma^{\mu}-\hat{q}^{\mu} \not{q}, \quad P_{2}^{\mu}=-i \sigma^{\mu \hat{q}},
$$

with $\sigma^{\mu \hat{q}} \equiv \sigma^{\mu \nu} \hat{q}_{\nu}$, hatted quantities are understood to be divided by $m_{\Lambda_{c}}$ (i.e. $\hat{q} \equiv q / m_{\Lambda_{c}}$ ), $\bar{u} \equiv \bar{u}\left(p_{p}, \sigma\right), u \equiv u\left(p, \lambda_{c}\right)$ and we define the amendment $p\left(\frac{1}{2}^{+}\right) \rightarrow N(1535)\left(\frac{1}{2}^{-}\right)$by $\bar{u} \rightarrow \bar{u} \gamma_{5}$ on the right-hand side. The introduction of

$$
f_{1}\left(q^{2}\right) \equiv \frac{\hat{f}_{1}\left(q^{2}\right)}{\hat{q}^{2}}
$$

will prove useful for implementing the regularity constraint. 
Since $q \cdot P_{1,2}=0, f_{0}$ is the scalar form factor. The non-vanishing scalar matrix elements are given by

$$
\begin{aligned}
& \left(m_{c}-m_{u}\right)\left\langle p_{J_{P}=\frac{1}{2}}+\left(p_{p}, \sigma\right)|\bar{u} c| \Lambda_{c}\left(p, \lambda_{c}\right)\right\rangle=\left(m_{\Lambda_{c}}-m_{p}\right) \bar{u} u f_{0}^{V}\left(q^{2}\right), \\
& \left(m_{c}+m_{u}\right)\left\langle N_{J_{P}=\frac{1}{2}}-\left(p_{p}, \sigma\right)\left|\bar{u} \gamma_{5} c\right| \Lambda_{c}\left(p, \lambda_{c}\right)\right\rangle=\left(m_{\Lambda_{c}}+m_{p}\right) \bar{u} \gamma_{5} u f_{0}^{A}\left(q^{2}\right) .
\end{aligned}
$$

There are further constraints as for the $B \rightarrow V$ form factors. The analogue of $A_{0}^{B \rightarrow V}(0)=$ $A_{3}^{B \rightarrow V}(0)$ and $T_{1}^{B \rightarrow V}(0)=T_{2}^{B \rightarrow V}(0)$ are $f_{0}^{V(A)}(0)=\hat{f}_{1}^{V(A)}(0)$ and $t_{2}^{V}(0)=t_{2}^{A}(0)$ respectively. The former assures regularity and the second one is an algebraic constraint that follows from Dirac algebra cf. appendix B.1. Summarising one finds the following constraints

$$
\frac{1}{2}^{+} \rightarrow \frac{1}{2}^{ \pm}: f_{0}^{V(A)}(0)=\hat{f}_{1}^{V(A)}(0), \quad t_{2}^{V}(0)=t_{2}^{A}(0),
$$

on the form factors which must be automatically satisfied by any computation. This makes up a total of ten independent form factors with three additional constraints at $q^{2}=0$.

\subsubsection{The $\Lambda_{J^{P}=\frac{1}{2}}{ }^{+} \rightarrow \Lambda_{J^{P}=\frac{3}{2}}^{*}$ form factors}

The discussion of the excited baryon form factors proceeds very much in analogy to section 3.1.1. The main difference is that there's an additional structure and this gives four additional form factors. For the $N(1535)\left(J^{P}=\frac{3}{2}^{-}\right)$final state, we define dimensionless form factors by ${ }^{5}$

$$
\begin{aligned}
\left\langle N\left(p_{p}, \sigma\right)\left|O_{V(A)}^{\mu}\right| \Lambda_{c}\left(p, \lambda_{c}\right)\right\rangle & =\bar{\Psi}_{\alpha}\left(\gamma_{5}\right)\left[\mathcal{P}_{1}^{\alpha \mu} F_{1}^{V(A)}+\mathcal{P}_{2}^{\alpha \mu} F_{2}^{V(A)}+\mathcal{P}_{3}^{\alpha \mu} F_{3}^{V(A)}+\mathcal{P}_{0}^{\alpha \mu} F_{0}^{V(A)}\right] u \\
\left\langle N\left(p_{p}, \sigma\right)\left|O_{T_{(5)}}^{\mu}\right| \Lambda_{c}\left(p, \lambda_{c}\right)\right\rangle & =\bar{\Psi}_{\alpha}\left(\gamma_{5}\right)\left[\mathcal{P}_{1}^{\alpha \mu} T_{1}^{V(A)}+\mathcal{P}_{2}^{\alpha \mu} T_{2}^{V(A)}+\mathcal{P}_{3}^{\alpha \mu} T_{3}^{V(A)}\right] u
\end{aligned}
$$

where the amendment $N(1535)\left(J^{P}=\frac{3}{2}^{-}\right) \rightarrow N(1720)\left({J^{P}}^{-} \frac{3}{2}^{+}\right)$is defined by $\bar{\Psi} \rightarrow \bar{\Psi} \gamma_{5}$ on the right-hand side. Above $F_{i} \equiv F_{i}\left(q^{2}\right), T_{i} \equiv T_{i}\left(q^{2}\right), u \equiv u\left(p, \lambda_{c}\right) \Psi_{\alpha} \equiv \Psi_{\alpha}\left(p_{N}, \sigma\right)$ is a $\frac{3}{2}$-spinor where $\alpha$ is a Lorentz index, satisfying $\gamma^{\alpha} \Psi_{\alpha}=0$ and $p_{N}^{\alpha} \Psi_{\alpha}=0$ cf. appendix A.3. The Lorentz structures $\mathcal{P}_{i}$ read

$$
\begin{array}{ll}
\mathcal{P}_{0}^{\alpha \mu}=\frac{\hat{q}^{\alpha} \hat{q}^{\mu}}{\hat{q}^{2}}, & \mathcal{P}_{1}^{\alpha \mu}=\hat{q}^{\alpha} \hat{p}_{N}^{\mu}-\left(\hat{p}_{N} \cdot \hat{q}\right) g^{\alpha \mu}, \\
\mathcal{P}_{2}^{\alpha \mu}=\hat{q}^{\alpha} \gamma^{\mu}-\not q^{\alpha \mu}, & \mathcal{P}_{3}^{\alpha \mu}=\hat{q}^{2} g^{\alpha \mu}-\hat{q}^{\alpha} \hat{q}^{\mu},
\end{array}
$$

where $\hat{p}_{N} \cdot \hat{q}=\frac{1}{2}\left(1-\hat{m}_{N}^{2}-\hat{q}^{2}\right)$. Similar to before since $q_{\mu} \mathcal{P}_{1,2,3}^{\alpha \mu}=0, F_{0}$ is the scalar form factor. The non-vanishing scalar matrix elements are

$$
\begin{aligned}
\left(\hat{m}_{c}-\hat{m}_{u}\right)\left\langle N_{J_{P}=\frac{3}{2}}+\left(p_{N}, \sigma\right)|\bar{u} c| \Lambda_{c}\left(p, \lambda_{c}\right)\right\rangle & =\hat{p} \cdot \bar{\Psi} u F_{0}^{V}\left(q^{2}\right), \\
\left(\hat{m}_{c}+\hat{m}_{u}\right)\left\langle N_{J_{P}=\frac{3}{2}}-\left(p_{N}, \sigma\right)\left|\bar{u} \gamma_{5} c\right| \Lambda_{c}\left(p, \lambda_{c}\right)\right\rangle & =\hat{p} \cdot \bar{\Psi} \gamma_{5} u F_{0}^{A}\left(q^{2}\right) .
\end{aligned}
$$

There are further constraints as for the $B \rightarrow V$ form factors. The regularity constraint at $q^{2}=0$ is $F_{0}^{A(V)}(0)=\hat{F}_{3}^{A(V)}(0)$ with the hatted form factor analogous as in (3.3),

\footnotetext{
${ }^{5}$ Contraction with $q$ does not lead to any loss of information at the level of the helicity amplitudes as there are only six of them cf. footnote 4. How this works out precisely has been elucidated recently in [38].
} 
and the analogue of $T_{1}^{B \rightarrow V}(0)=T_{2}^{B \rightarrow V}(0)$ is the double constraint $T_{1}^{V}(0)=T_{1}^{A}(0)$ and $T_{2}^{A}(0)=T_{2}^{V}(0)+\hat{m}_{N} T_{1}^{V}(0)$ which we have derived using all the seven Lorentz structures (cf. appendix B.2) and thereby go beyond the current literature. This makes a total of fourteen independent form factors with four additional constraint at $q^{2}=0$. In summary the following constraints

$$
\frac{1}{2}^{+} \rightarrow \frac{3}{2}^{\mp}: F_{0}^{V(A)}(0)=\hat{F}_{3}^{V(A)}(0), T_{1}^{V}(0)=T_{1}^{A}(0), T_{2}^{A(V)}(0)=T_{2}^{V(A)}(0)+\hat{m}_{N} T_{1}^{V(A)}(0),
$$

apply and must hold independent of the method of computation.

\subsection{The baryonic helicity amplitudes and verification of endpoint relations}

In this section we give the explicit HAs in terms of form factors. Being more explicit than previously, the HAs read

$$
\begin{aligned}
& H_{\lambda_{q} \bar{\sigma}}^{V(A)} \equiv\left\langle p(N)\left(p_{p(N)}, \sigma\right)\left|O_{V(A)}^{\mu}\right| \Lambda_{c}\left(p, \lambda_{c}\right)\right\rangle \beta_{\mu}^{*}\left(q, \lambda_{q}\right) \\
& H_{\lambda_{q} \bar{\sigma}}^{T\left(T_{5}\right)} \equiv\left\langle p(N)\left(p_{p(N)}, \sigma\right)\left|O_{T_{(5)}}^{\mu}\right| \Lambda_{c}\left(p, \lambda_{c}\right)\right\rangle \beta_{\mu}^{*}\left(q, \lambda_{q}\right) .
\end{aligned}
$$

The following kinematic abbreviations are helpful

$$
\begin{aligned}
& \Delta_{ \pm} \equiv m_{\Lambda_{c}} \pm m_{p(N)}, \quad s_{ \pm} \equiv \Delta_{ \pm}^{2}-q^{2}, \\
& \lambda\left(m_{\Lambda_{c}}^{2}, m_{p(N)}^{2}, q^{2}\right)=s_{+} s_{-}=4 m_{\Lambda_{c}}^{2} \kappa, \quad \Delta^{2}=m_{\Lambda_{c}}^{2}-m_{p(N)}^{2},
\end{aligned}
$$

where $\lambda$ is the Källén-function, defined above (2.7). Moreover, the substitutions at the kinematic endpoint are as follows

$$
q^{2} \rightarrow \Delta_{-}^{2}, \quad \sqrt{s_{+}} \rightarrow 2 \sqrt{m_{\Lambda_{c}} m_{p(N)}}, \quad \sqrt{s_{-}} \rightarrow \sqrt{\frac{m_{\Lambda_{c}}}{m_{p(N)}}} \kappa .
$$

\subsubsection{The $\Lambda_{J^{P}=\frac{1}{2}}{ }^{+} \rightarrow \Lambda_{J^{P}=\frac{1}{2}}^{\prime}{ }^{ \pm}$helicity amplitudes}

Using the explicit form factor parameterisation in (3.1), for $\frac{1}{2}^{+} \rightarrow \frac{1}{2}^{+}$, we get the following expressions

$$
\begin{aligned}
& \hat{H}_{0 \frac{1}{2}}^{V(A)}=\sqrt{\hat{q}^{2} \hat{s}_{\mp}}\left(\hat{\Delta}_{ \pm} f_{1}^{V(A)}+f_{2}^{V(A)}\right) \stackrel{\text { endpoint }}{\rightarrow}\left\{\begin{array}{c}
\vec{f} \cdot \vec{v}_{0 \frac{1}{2}} c_{1 / 2} \hat{\kappa} \\
\hat{E}_{1 / 2}^{A}
\end{array}\right. \\
& \hat{H}_{1 \frac{1}{2}}^{V(A)}=\sqrt{2 \hat{s}_{\mp}}\left(\hat{q}^{2} f_{1}^{V(A)}+\hat{\Delta}_{ \pm} f_{2}^{V(A)}\right) \rightarrow\left\{\begin{array}{l}
-\sqrt{2} \vec{f} \cdot \vec{v}_{1 \frac{1}{2}} c_{1 / 2} \hat{\kappa} \\
-\sqrt{2} \hat{E}_{1 / 2}^{A}
\end{array}\right.
\end{aligned}
$$

for the vector and the axial HAs, evaluated to leading order in $\kappa$ on the right-hand side at the kinematic endpoint with

$$
\hat{E}_{1 / 2}^{A} \equiv 2 \hat{\Delta}_{-} \sqrt{\hat{m}_{p}}\left(\hat{\Delta}_{-} f_{1}^{A}\left(\Delta_{-}^{2}\right)+f_{2}^{A}\left(\Delta_{-}^{2}\right)\right)
$$


the auxiliary velocity vectors read $\left(c_{1 / 2} \equiv 1 / \sqrt{\hat{m}_{p}}\right)$

$$
\vec{v}_{0 \frac{1}{2}}=\left(\hat{\Delta}_{+} \hat{\Delta}_{-}, \hat{\Delta}_{-}\right), \quad \vec{v}_{1 \frac{1}{2}}=-\left(\hat{\Delta}_{-}^{2}, \hat{\Delta}_{+}\right),
$$

and the scalar product is to be interpreted as a two dimensional one in Euclidean space with $\vec{f}=\left(f_{1}, f_{2}\right)$. For the timelike HA we obtain

$$
\hat{H}_{t \frac{1}{2}}^{V(A)}= \pm \sqrt{\frac{\hat{s}_{ \pm}}{\hat{q}^{2}}} \hat{\Delta}_{\mp} f_{0}^{V(A)} \rightarrow\left\{\begin{array}{l}
2 \sqrt{\hat{m}_{p}} f_{0}^{V}\left(\Delta_{-}\right) \\
-\frac{1}{\sqrt{\hat{m}_{p}}} \frac{\hat{\Delta}_{+}}{\hat{\Delta}_{-}} f_{0}^{A}\left(\Delta_{-}\right) \hat{\kappa}
\end{array} .\right.
$$

All other HAs follow by substitution rules from the ones above. Firstly, the tensor amplitudes follow from the vector amplitudes by replacing $\left(f_{0}, f_{1}, f_{2}\right) \rightarrow\left(0, t_{1}, t_{2}\right)$. Second,

$$
J^{P}=\frac{1}{2}^{+}: \quad H_{\bar{\lambda}_{q} \bar{\sigma}}^{V(A)}= \pm H_{\lambda_{q} \sigma}^{V(A)}, \quad J^{P}=\frac{1}{2}^{-}: \quad H_{\bar{\lambda}_{q} \bar{\sigma}}^{V(A)}=\mp H_{\lambda_{q} \sigma}^{V(A)} .
$$

Third, the $\frac{1}{2}^{+} \rightarrow \frac{1}{2}^{-}$HA are obtained by replacing $f_{i}^{V(A)}, t_{i}^{V(A)}$ by $f_{i}^{A(V)}, t_{i}^{A(V)}$ which follows from the definition (3.1). At last we observe that the endpoint relations (2.9),

$$
\hat{H}_{0 \frac{1}{2}}^{A\left(T_{5}\right)}=-\frac{1}{\sqrt{2}} \hat{H}_{1 \frac{1}{2}}^{A\left(T_{5}\right)}
$$

is obeyed as dictated by the Wigner-Eckart theorem.

\subsubsection{The $\Lambda_{J^{P}=\frac{1}{2}}{ }^{+} \rightarrow \Lambda_{J^{P}=\frac{3}{2}}^{*}$ helicity amplitudes}

Employing the form factor parameterisation in (3.6), for $\frac{1}{2}^{+} \rightarrow \frac{3}{2}^{-}$, the following expressions are obtained

$$
\begin{aligned}
& \hat{H}_{0 \frac{1}{2}}^{V(A)}= \pm \frac{\sqrt{2 \hat{q}^{2} \hat{s}_{ \pm}}}{\sqrt{3}}\left(\left( \pm \hat{m}_{N}\right) F_{1}^{V(A)}+F_{2}^{V(A)}+\frac{q^{2}-\hat{\Delta}^{2}}{2 \sqrt{2}\left( \pm \hat{m}_{N}\right)} F_{3}^{V(A)}\right) \rightarrow\left\{\begin{array}{c}
\hat{E}_{3 / 2}^{V} \\
\vec{F} \cdot \vec{v}_{0 \frac{1}{2}} c_{3 / 2} \hat{\kappa}
\end{array},\right. \\
& \hat{H}_{1 \frac{1}{2}}^{V(A)}=\frac{\sqrt{\hat{s}_{ \pm}}}{\sqrt{3}}\left(\frac{q^{2}-\hat{\Delta}^{2}}{2} F_{1}^{V(A)}+\frac{q^{2}-\hat{\Delta}_{\mp}}{\left( \pm \hat{m}_{N}\right)} F_{2}^{V(A)}+\hat{q}^{2} F_{3}^{V(A)}\right) \quad \rightarrow\left\{\begin{array}{l}
-\sqrt{\frac{1}{2}} \hat{E}_{3 / 2}^{V} \\
-\sqrt{\frac{1}{2}} \vec{F}_{v_{1} \frac{1}{2}} c_{3 / 2} \hat{\kappa}
\end{array},\right. \\
& \hat{H}_{\overline{1} \frac{3}{2}}^{V(A)}=\sqrt{\hat{s}_{+}}\left(\frac{\hat{q}^{2}-\hat{\Delta}^{2}}{2} F_{1}^{V(A)}-\hat{\Delta}_{\mp} F_{2}^{V(A)}+\hat{q}^{2} F_{3}^{V(A)}\right) \quad \rightarrow\left\{\begin{array}{l}
-\sqrt{\frac{3}{2}} \hat{E}_{3 / 2}^{V} \\
-\sqrt{\frac{3}{2}} \vec{F} \cdot \vec{v}_{\overline{1} \frac{3}{2}} c_{3 / 2} \hat{\kappa}
\end{array},\right.
\end{aligned}
$$

where the endpoint expression reads

$$
\hat{E}_{3 / 2}^{V} \equiv \sqrt{\frac{8 \hat{m}_{N}}{3}} \hat{\Delta}_{-}\left(\hat{m}_{N} F_{1}^{V}\left(\Delta_{-}^{2}\right)+F_{2}^{V}\left(\Delta_{-}^{2}\right)-\hat{\Delta}_{-} F_{3}^{V}\left(\Delta_{-}^{2}\right)\right),
$$

the auxiliary velocity vectors are $\left(c_{3 / 2} \equiv \frac{\sqrt{2} \hat{\Delta}_{-}}{\sqrt{3 \hat{m}_{N}}}\right)$

$$
\vec{v}_{0 \frac{1}{2}}=\left(\hat{m}_{N},-1,-\hat{\Delta}_{-}\right), \quad \vec{v}_{1 \frac{1}{2}}=\left(\hat{m}_{N},-\frac{3+\hat{m}_{N}}{1-\hat{m}_{N}},-\hat{\Delta}_{-}\right), \quad \vec{v}_{\overline{1}_{\frac{3}{2}}}=\left(\hat{m}_{N}, \frac{1+\hat{m}_{N}}{1-\hat{m}_{N}},-\hat{\Delta}_{-}\right),
$$


and the scalar product is to be interpreted as for the $\frac{1}{2}$-case. For the timelike HA we obtain

$$
\hat{H}_{t \frac{1}{2}}^{V(A)}=-\sqrt{\frac{\hat{s}_{\mp}}{6 \hat{q}^{2}}} \frac{\hat{s}_{ \pm}}{\hat{m}_{N}} F_{0}^{V(A)} \rightarrow\left\{\begin{array}{l}
-2 \sqrt{\frac{2}{3}} \frac{1}{\hat{m}_{N}^{1 / 2} \hat{\Delta}_{-}} F_{0}^{V}\left(\Delta_{-}^{2}\right) \hat{\kappa} \\
-\sqrt{\frac{2}{3}} \frac{1}{\hat{m}_{N}^{3 / 2} \hat{\Delta}_{-}} F_{0}^{A}\left(\Delta_{-}^{2}\right) \hat{\kappa}^{2}
\end{array} .\right.
$$

The substitution rules for the other HAs are as follows. Firstly, the tensor amplitudes follow from the vector amplitudes by replacing $\left(F_{0}, F_{1}, F_{2}, F_{3}\right) \rightarrow\left(0, T_{1}, T_{2}, T_{3}\right)$. Second,

$$
J^{P}=\frac{3}{2}^{-}: \quad H_{\bar{\lambda}_{q} \bar{\sigma}}^{V(A)}= \pm H_{\lambda_{q} \sigma}^{V(A)}, \quad J^{P}=\frac{3}{2}^{+}: \quad H_{\bar{\lambda}_{q} \bar{\sigma}}^{V(A)}=\mp H_{\lambda_{q} \sigma}^{V(A)} .
$$

Third, the $\frac{1}{2}^{+} \rightarrow \frac{3}{2}^{+}$HA are obtained by the replacement $F_{i}^{V(A)}, T_{i}^{V(A)} \rightarrow F_{i}^{A(V)}, T_{i}^{A(V)}$. The endpoint relations $(2.10)$,

$$
\hat{H}_{0 \frac{1}{2}}^{V(T)}=-\sqrt{2} \hat{H}_{1 \frac{1}{2}}^{V(T)}=-\sqrt{\frac{2}{3}} \hat{H}_{\overline{1} \frac{3}{2}}^{V(T)}
$$

are satisfied by the explicit expression in (3.19).

\subsection{Specific endpoint features compared with $B \rightarrow V \ell_{1} \bar{\ell}_{2}$}

In this section we compare the endpoint behaviour of the $\frac{1}{2}$ - and $\frac{3}{2}$-HAs, of the previous section, with the ones for $B \rightarrow V \ell_{1} \bar{\ell}_{2}$. Generally, parity determines whether the power of the velocity $\kappa$ is even or odd. This is the appeal of the so-called

$$
H_{\perp(\|)}=\frac{1}{\sqrt{2}}\left(H_{1} \mp H_{\overline{1}}\right), \quad H_{0}=H_{0},
$$

transversity basis (the subscript on the right-hand side corresponds to the helicity index $\lambda_{q}$ ). Now the $H_{\perp, \|, t}$ HAs, but not $H_{0}$, are of definite parity and this leads to selection rules in the power of the velocity collected in table 1 for the two types baryonic modes compared against $0^{-} \rightarrow 1^{ \pm}$which includes $B \rightarrow V \ell_{1} \bar{\ell}_{2}$ discussed in our previous work.

An interesting feature is that in the $\frac{1}{2} \rightarrow \frac{1}{2}$ mode the timelike HA (3.16) does not vanish at the endpoint unlike in the other cases. The reason for this is that the mode can be in an $S$-wave $(l=0)$, w.r.t. the lepton pair, whereas the other ones require a $P$-wave $(l=1)$. For most of phenomenology this term however remains unimportant since it is suppressed by lepton masses.

In the context of $B \rightarrow V \ell_{1} \bar{\ell}_{2}$ we advocated a $\kappa$-expansion which was based on the universality of terms linear in $\kappa$. Inspecting the results in eqs. (3.13) and (3.19) one observes that this universality no longer holds for baryons. The reason for it is that there are several form factors for each parity. This is in contradistinction to $B \rightarrow V \ell_{1} \bar{\ell}_{2}$ where the vector operator $O_{V}^{\mu}(2.1)$ gives rise to a single form factor, denoted by $V^{B \rightarrow V}\left(q^{2}\right)$. Hence in the baryonic case the form factor dependence does not drop when ratios of HAs, linear in $\kappa$, are taken. Hence in the baryonic case the form factor dependence does not drop when ratios of HAs, linear in $\kappa$, are taken. From a group theoretical viewpoint there is no unique invariant in these cases since the relevant Kronecker product, $\frac{1}{2} \otimes \frac{1}{2}\left(\frac{3}{2}\right) \otimes \mathbf{1} \otimes \mathbf{1}=\mathbf{2} \cdot \mathbf{0}+\ldots$ cf. [1] section 2.2, contains two invariants. From these viewpoints the $\kappa$-expansion in $B \rightarrow V \ell_{1} \bar{\ell}_{2}$ might be seen as somewhat accidental. 


\begin{tabular}{|l|l|l|lc|l|}
\hline$J^{P} \rightarrow{J^{\prime}}^{P^{\prime}}$ & $H_{\perp}$ & $H_{\|}$ & $H_{0}$ & $H_{t}$ \\
\hline$\frac{1}{2}^{+} \rightarrow \frac{1}{2}^{ \pm}$ & $H^{A(V)} \propto \kappa^{0(1)}$ & $H^{V(A)} \propto \kappa^{1(0)}$ & $H^{V} \propto \kappa^{1(0)}$ & $H^{A} \propto \kappa^{0(1)}$ & $H^{V(A)} \propto \kappa^{0(0)}$ \\
$\frac{1}{2}^{+} \rightarrow \frac{3}{2}^{\mp}$ & $H^{A(V)} \propto \kappa^{1(0)}$ & $H^{V(A)} \propto \kappa^{0(1)}$ & $H^{V} \propto \kappa^{0(1)}$ & $H^{A} \propto \kappa^{1(0)}$ & $H^{V(A)} \propto \kappa^{1(1)}$ \\
$0^{-} \rightarrow 1^{ \pm}$ & $H^{A(V)} \propto \kappa^{0(1)}$ & $H^{V(A)} \propto \kappa^{1(0)}$ & $H^{V(A)} \propto \kappa^{1(0)}$ & & $H^{V(A)} \propto \kappa^{1(1)}$ \\
\hline
\end{tabular}

Table 1. Leading $\kappa$-dependance in $J^{P} \rightarrow J^{P^{\prime}}$ HAs. The table is an extension of table 1 in [2] where $C_{ \pm}$corresponds to $H^{V(A)}$ for $0^{-} \rightarrow 1^{-}$which was the main focus of that paper. Changing the parity of the initial state demands $V \leftrightarrow A$ in the table above. HAs which are not given, such as $\left.H_{\perp}^{A}\right|_{\frac{1}{2}+\rightarrow \frac{1}{2}^{-}}$, do vanish by parity in QCD. The HA $H_{0}$ is not of definite parity and this is why both vector and axial ones contributes to the same power. The $T\left(T_{5}\right)$ HAs follows the same pattern as the ones for $V(A)$ respectively.

\section{Constraints on helicity based form factors}

Before applying our results to particular angular distributions in the literature we are inclined to clarify a point which does not seem to have received specific attention in the literature. ${ }^{6}$ This concerns constraints on helicity-based form factors which are often used when discussing specific angular distributions e.g. [40] and [37].

The helicity-based form factors have been introduced in [41] for the $\frac{1}{2}^{+} \rightarrow \frac{1}{2}^{+}$case and extended to $\frac{1}{2} \rightarrow \frac{3}{2}$ in references [37, 42]. The basic idea is that each HA is proportional to a single form factor. This of course raises the question of how the endpoint relations (2.9) and (2.10) can be satisfied. This can only be remedied if there are constraints between the helicity form factors. This is indeed the case as the price for introducing helicity-based form factors is that the analytic structure of the form factors and the HAs differ. This is due to $\frac{1}{s_{ \pm}}$-poles in the analogue of the Lorentz structures (3.2) and (3.7). Now, since by first principles there cannot be any poles of the form $\frac{1}{s_{ \pm}}$in the HA, we are to conclude that there must be constraints. ${ }^{7}$

For completeness and clarity we reproduce the helicity-based form factor definition [41], an alternative to (3.1), but using the notation in [40] in section $3^{8}$ The constraint for the $\frac{1}{2} \rightarrow \frac{1}{2}$-case has been derived in [46] indirectly by using a similar basis to ours and requiring

\footnotetext{
${ }^{6}$ The discussion in [37] has not been worked out into concrete constraints. Additionally, in our opinion, the dependence on $s_{ \pm}$from the spinors should not enter the discussion as they are external objects to the correlation function.

${ }^{7} \mathrm{~A}$ pole would correspond to a particle of mass $\Delta_{ \pm}^{2}$ by the dispersion relation or LSZ formalism e.g. chapter 10 in [43]. Note though, that for there can be pole singularities at $q^{2}=\Delta_{+}^{2}$ in the form factor. There can only but branch cuts, corresponding to the threshold of the $\ell^{+} \ell^{-} \rightarrow \Lambda_{c} p(N)$ process related by crossing symmetry. At $q^{2}=\Delta_{-}^{2}$ there are no singularities of this type on the first sheet but there are branch cuts on the second sheet. These type of singularities are known as pseudo-thresholds [44]. For a simple example thereof we refer the reader to the lectures notes [45]. In summary whereas there are singularities at $\Delta_{ \pm}$, in terms of branch cuts, pole singularities are absent because there is no known (stable) particle in QCD of this mass and quantum numbers.

${ }^{8}$ The definition is more straightforward in [41] but a change of notation is was undertaken from [41] to [40]: $f(g)_{+}=f_{0}^{V(A)}$ and $f(g)_{\perp}=f_{\perp}^{V(A)}$.
} 
them to match with the helicity form factor based results.

$$
\begin{aligned}
\left\langle p\left(p_{p}, \sigma\right)\left|O_{V(A)}^{\mu}\right| \Lambda_{c}\left(p, \lambda_{c}\right)\right\rangle & = \pm \bar{u}\left(\gamma_{5}\right)\left[Q_{0}^{\mu} f_{0}^{V(A)}\left(q^{2}\right)+Q_{\perp}^{\mu} f_{\perp}^{V(A)}\left(q^{2}\right)+Q_{t}^{\mu} f_{t}^{V(A)}\left(q^{2}\right)\right] u, \\
\left\langle p\left(p_{p}, \sigma\right)\left|O_{T_{(5)}}^{\mu}\right| \Lambda_{c}\left(p, \lambda_{c}\right)\right\rangle & = \pm \bar{u}\left(\gamma_{5}\right)\left[Q_{0}^{\mu} f_{0}^{T\left(T_{5}\right)}\left(q^{2}\right)+Q_{\perp}^{\mu} f_{\perp}^{T\left(T_{5}\right)}\left(q^{2}\right)\right] u
\end{aligned}
$$

where the Lorentz structures are

$$
Q_{t}^{\mu}=\Delta_{\mp} \frac{q_{\mu}}{q^{2}}, \quad Q_{0}^{\mu}=\frac{\Delta_{ \pm}}{s_{ \pm}}\left(p+p_{p}-\frac{\Delta^{2}}{q^{2}} q\right)^{\mu}, \quad Q_{\perp}^{\mu}=\left(\gamma \mp \frac{2 m_{p}}{s_{ \pm}} p_{p}-\frac{2 m_{\Lambda_{c}}}{s_{ \pm}} p\right)^{\mu},
$$

with abbreviation defined in (3.11) and with some mild abuse of notation the first and second entry correspond to $V, T$ and $A, T_{5}$ respectively. Now, by requiring the absence of poles at $q^{2}=\Delta_{-}^{2} \equiv q_{\max }^{2}$ one find the following constraints

$$
\frac{1}{2}^{+} \rightarrow \frac{1}{2}^{ \pm}: f_{0}^{A(V)}\left(q_{\max }^{2}\right)=f_{\perp}^{A(V)}\left(q_{\max }^{2}\right)
$$

All results in this section are valid for the tensor form factors as well with the usual replacements $V, A \rightarrow T, T_{5}$. The further constraint at $q^{2}=\Delta_{+}^{2}$ reads

$$
\frac{1}{2}^{+} \rightarrow \frac{1}{2}^{ \pm}: f_{0}^{V(A)}\left(\Delta_{+}^{2}\right)=f_{\perp}^{V(A)}\left(\Delta_{+}^{2}\right)
$$

and can be obtained by interchanging $V \leftrightarrow A$ and $m_{p} \leftrightarrow-m_{p}$ from the one at $q^{2}=\Delta_{-}^{2}$. Applying (4.3) to the expressions in eq. 3.13 in [40] one does indeed find that $H_{0}^{A}(+1 / 2,+1 / 2)$ : $H_{+}^{A}(-1 / 2,+1 / 2)=1:-\sqrt{2}$ in agreement with our generic prediction (2.9). ${ }^{9}$

Again, for completeness and clarity we reproduce the $\frac{1}{2} \rightarrow \frac{3}{2}$ helicity-based form factors, using notation in [37] except introducing the dimensionless form factor $\bar{f}_{g}=f_{g} / m_{\Lambda_{c}}$,

$$
\begin{aligned}
\left\langle N\left(p_{p}, \sigma\right)\left|O_{V(A)}^{\mu}\right| \Lambda_{c}\left(p, \lambda_{c}\right)\right\rangle & =\bar{\Psi}_{\alpha}\left(\gamma_{5}\right)\left[p^{\alpha}\left(Q_{0}^{\mu} f_{0}^{V(A)}+Q_{\perp}^{\mu} f_{\perp}^{V(A)}+Q_{t}^{\mu} f_{t}^{V(A)}\right)+Q_{g}^{\alpha \mu} \bar{f}_{g}^{V(A)}\right] u, \\
\left\langle N\left(p_{p}, \sigma\right)\left|O_{T_{(5)}}^{\mu}\right| \Lambda_{c}\left(p, \lambda_{c}\right)\right\rangle & =\bar{\Psi}_{\alpha}\left(\gamma_{5}\right)\left[p^{\alpha}\left(Q_{0}^{\mu} f_{0}^{T\left(T_{5}\right)}+Q_{\perp}^{\mu} f_{\perp}^{T\left(T_{5}\right)}+Q_{g}^{\alpha \mu} \bar{f}_{g}^{T\left(T_{5}\right)}\right] u,\right.
\end{aligned}
$$

with $Q^{\mu}$ as defined in (4.2) and

$$
Q_{g}^{\alpha \mu}=m_{\Lambda_{c}}\left(g^{\alpha \mu}-\frac{m_{N} p^{\alpha}}{s_{\mp}}\left(\gamma \mp \frac{2 p_{p}}{m_{N}} \pm 2 \frac{m_{N} p \pm m_{\Lambda_{c}} p_{p}}{s_{ \pm}}\right)^{\mu}\right),
$$

with the same meaning for the first and second entry as previously. Proceeding along the same reasoning as before, one gets the following constraints at $q^{2}=\Delta_{-}^{2} \equiv q_{\max }^{2}$

$$
\begin{aligned}
\frac{1}{2}^{+} \rightarrow \frac{3}{2}^{\mp}: \quad f_{\perp}^{V(A)}\left(q_{\max }^{2}\right) & =-\frac{m_{\Lambda_{c}} m_{N} \bar{f}_{g}^{V(A)}\left(q_{\max }^{2}\right)}{s_{-}} \\
f_{0}^{V(A)}\left(q_{\max }^{2}\right) & =\frac{2 m_{\Lambda_{c}} m_{N} \bar{f}_{g}^{V(A)}\left(q_{\max }^{2}\right)}{s_{-}} \frac{m_{\Lambda_{c}}-m_{N}}{m_{\Lambda_{c}}+m_{N}} \\
f_{\perp}^{A(V)}\left(q_{\max }^{2}\right) & =f_{0}^{A(V)}\left(q_{\max }^{2}\right)-\frac{\bar{f}_{g}^{A(V)}\left(q_{\max }^{2}\right)}{4}
\end{aligned}
$$

\footnotetext{
${ }^{9}$ We do not seek to find agreement in signs since this depends on the conventions of the authors. Of course these sign cancel when combined with the specific angular decay rates, provided everything was handled consistently. Nevertheless or somewhat accidentally signs agree.
} 
Since $\bar{f}_{g}\left(q_{\text {max }}^{2}\right)$ is generically non-vanishing this means that the $f_{0, \perp}$ form factors are singular at the endpoint and can be seen as a justification for introducing the slightly different notation as in [42]. The constraint at $q^{2}=\Delta_{+}^{2}$ reads

$$
\begin{aligned}
\frac{1}{2}^{+} \rightarrow \frac{3}{2}^{\mp}: f_{\perp}^{A(V)}\left(\Delta_{+}^{2}\right) & =\frac{m_{\Lambda_{c}} m_{N} \bar{f}_{g}^{A(V)}\left(\Delta_{+}^{2}\right)}{s_{+}}, \\
f_{0}^{A(V)}\left(\Delta_{+}^{2}\right) & =-\frac{2 m_{\Lambda_{c}} m_{N} \bar{f}_{g}^{A(V)}\left(\Delta_{+}^{2}\right)}{s_{+}} \frac{m_{\Lambda_{c}}+m_{N}}{m_{\Lambda_{c}}-m_{N}}, \\
f_{\perp}^{V(A)}\left(\Delta_{+}^{2}\right) & =f_{0}^{V(A)}\left(\Delta_{+}^{2}\right)-\frac{\bar{f}_{g}^{V(A)}\left(\Delta_{+}^{2}\right)}{4}
\end{aligned}
$$

and can again be obtained by interchanging $V \leftrightarrow A$ and $m_{N} \leftrightarrow-m_{N}$. Applying (4.7) to eq. 3.8 in [37] we do find that $H_{0}^{V}(+1 / 2,+1 / 2): H_{+}^{V}(1 / 2,-1 / 2): H_{+}^{V}(1 / 2,3 / 2)=1:-\sqrt{\frac{1}{2}}$ : $-\sqrt{\frac{3}{2}}$ in accordance with $(2.10)$. In summary, as expected, once the constraints are applied the endpoint relations are satisfied.

\subsection{Comparison with the literature}

From the viewpoint of theory endpoint relations of HAs are valuable for consistency checks of computations and valid fit parameterisations in connections with form factor constraints. The concrete use for experiment is discussed in the upcoming section 5.3.

Since we have clarified how endpoint relations work it seems worthwhile to mention a few papers that could benefit from applying this knowledge for checks and parameterisations. For example, the results in [47] for both $\frac{1}{2}^{+} \rightarrow \frac{1}{2}^{+}$and $\frac{1}{2}^{+} \rightarrow \frac{3}{2}^{-}$do not satisfy the endpoint relations (even when the subleading Isgur-Wise function is set to zero). There are minor issues in [48] fo $\Lambda_{b} \rightarrow \Sigma$. The lattice form factor fit for $\Xi_{c} \rightarrow \Xi$ [12] needs the constraints (4.3) imposed in order to satisfy the endpoint relations. We do agree with the $\frac{1}{2}^{+} \rightarrow \frac{3}{2}^{-}$results in [37] and the endpoint relation are satisfied once the constraints (4.7) are imposed.

\section{Angular distributions}

We provide a brief analysis of angular distributions in the limit of zero lepton mass based on the references [40] and [37] for the $\frac{1}{2}^{+} \rightarrow \frac{1}{2}^{+}$and $\frac{1}{2}^{+} \rightarrow \frac{3}{2}^{-}$cases. ${ }^{10}$ We define the normalised distributions as follows

$$
\begin{aligned}
\hat{K}\left(q^{2}, \theta_{\ell}, \theta_{\Lambda}, \phi\right) & =\left(\frac{d \Gamma}{d q^{2}}\right)^{-1} \frac{d^{4} \Gamma\left(\Lambda_{b} \rightarrow \Lambda(\rightarrow N \pi) \ell^{+} \ell^{-}\right)}{d q^{2} d \cos \theta_{\ell} d \cos \theta_{\Lambda} d \phi} \\
\hat{L}\left(q^{2}, \theta_{\ell}, \theta_{\Lambda}^{*}, \phi\right) & =\left(\frac{d \Gamma}{d q^{2}}\right)^{-1} \frac{d^{4} \Gamma\left(\Lambda_{b} \rightarrow \Lambda(1520)(\rightarrow N \bar{K}) \ell^{+} \ell^{-}\right)}{d q^{2} d \cos \theta_{\ell} d \cos \theta_{\Lambda}^{*} d \phi},
\end{aligned}
$$

where we refer to the references above for the definition of the decay angles. In what follows we assume that the initial baryon is unpolarised. A salient difference between the two decays is that the $\Lambda$ is stable in QCD and decays via the weak decay $\Lambda \rightarrow N \pi$, parameterised by the parity-violating parameter $\alpha$. In what follows we introduce the notation $\left\langle f\left(\theta_{\ell}, \theta_{\Lambda}^{(*)}, \phi\right)\right\rangle$

$$
\left\langle f\left(\theta_{\ell}, \theta_{\Lambda}^{(*)}, \phi\right)\right\rangle=\int_{-1}^{1} d \cos \theta_{\ell} \int_{-1}^{1} d \cos \theta_{\Lambda}^{(*)} \int_{0}^{2 \pi} d \phi f\left(\theta_{\ell}, \theta_{\Lambda}^{(*)}, \phi\right)
$$

\footnotetext{
${ }^{10}$ The adaption to the other parity final states is relatively straightforward when taking into account the previously stated transformation rules.
} 
to stand for the averaging over the angles such that

$$
\begin{aligned}
\langle\hat{K}\rangle & =\frac{\Omega}{3}\left(\hat{K}_{1 c c}+2 \hat{K}_{1 s s}\right)=1, \\
\langle\hat{L}\rangle & =\frac{\Omega}{9}\left(\hat{L}_{1 c c}+2\left(\hat{L}_{1 s s}+\hat{L}_{2 c c}+2 \hat{L}_{2 s s}+\hat{L}_{3 s s}\right)\right)=1,
\end{aligned}
$$

are normalised to unity and $\Omega \equiv\langle 1\rangle=8 \pi$ is the phase space normalisation factor.

\subsection{The $\Lambda_{J^{P}=\frac{1}{2}}{ }^{+} \rightarrow \Lambda_{J^{P}=\frac{1}{2}}^{\prime}{ }^{ \pm}$angular distribution at the endpoint}

For the $\frac{1}{2}^{+} \rightarrow \frac{1}{2}^{+}$case we reproduce for convenience the distribution found in [40]

$$
\begin{aligned}
\hat{K}\left(q^{2}, \theta_{\ell}, \theta_{\Lambda}, \phi\right)= & \left(\hat{K}_{1 s s} \sin ^{2} \theta_{\ell}+\hat{K}_{1 c c} \cos ^{2} \theta_{\ell}+\hat{K}_{1 c} \cos \theta_{\ell}\right) \\
& +\left(\hat{K}_{2 s s} \sin ^{2} \theta_{\ell}+\hat{K}_{2 c c} \cos ^{2} \theta_{\ell}+\hat{K}_{2 c} \cos \theta_{\ell}\right) \cos \theta_{\Lambda} \\
& +\left(\hat{K}_{3 s c} \sin \theta_{\ell} \cos \theta_{\ell}+\hat{K}_{3 s} \sin \theta_{\ell}\right) \sin \theta_{\Lambda} \sin \phi \\
& +\left(\hat{K}_{4 s c} \sin \theta_{\ell} \cos \theta_{\ell}+\hat{K}_{4 s} \sin \theta_{\ell}\right) \sin \theta_{\Lambda} \cos \phi,
\end{aligned}
$$

for which $\Lambda_{b} \rightarrow \Lambda(\rightarrow N \pi) \ell^{+} \ell^{-}$was considered in specifically. The distribution including initial state polarisation can be found in [49]. All terms involving $\theta_{\Lambda}$ are then proportional to the parity-violating parameter

$$
\alpha=\frac{\left|H_{0 \frac{1}{2}}\right|^{2}-\left|H_{0 \frac{1}{2}}\right|^{2}}{\left|H_{0 \frac{1}{2}}\right|^{2}+\left|H_{0 \frac{1}{2}}\right|^{2}},
$$

and some values are known: e.g. $\alpha_{\Lambda \rightarrow p \pi^{-}}=0.732(14)$ [33]. Note that if the final state baryon decays via the strong force then $\alpha \rightarrow 0$. Using our results, the particular expressions found in section 4 , and inserting them into their expressions for the $K$ 's we find that at the endpoint

$$
\begin{aligned}
\left\{\hat{K}_{1 s s}, \hat{K}_{1 c c}\right\} & \rightarrow \frac{1}{\Omega}\{1,1\}, \\
\left\{\hat{K}_{2 c}, \hat{K}_{4 s}\right\} & \rightarrow \frac{\alpha r}{\Omega}\{-1,1\}, \quad r \approx \frac{2 \operatorname{Re}\left[\left(C_{9}-C_{9}^{\prime}\right)\left(C_{10}-C_{10}^{\prime}\right)^{*}\right]}{\left|C_{9}-C_{9}^{\prime}\right|^{2}+\left|C_{10}-C_{10}^{\prime}\right|^{2}},
\end{aligned}
$$

where other terms are vanishing because either the amplitudes in question vanish or they vanish as all HAs are proportional to each other. The latter point implies that the $K^{\prime} s$ proportional to imaginary parts of products of amplitudes vanish. The factor $r$, which receives additional correction from dipole operators which are however negligible in the Standard Model, is a curiosity of the parity violation in the decay which would not be present if the $\Lambda$ would decay via the strong force $(\alpha=0)$. Parity conservation of QCD assures that endpoint relations are more effective in constraining the angular distribution at the endpoint. Moreover, if the decay was adapted to $\frac{1}{2}^{+} \rightarrow \frac{1}{2}^{-}$then $C^{\prime} \rightarrow-C^{\prime}$ which makes it manifest that this is a parity violating channel. Finally the angular distribution in (5.4) assumes the simple form

$$
\hat{K}\left(q^{2}, \theta_{\ell}, \theta_{\Lambda}, \phi\right) \rightarrow \frac{1}{\Omega}\left(1-\alpha r\left(\cos \theta_{\ell} \cos \theta_{\Lambda}-\sin \theta_{\ell} \sin \theta_{\Lambda} \cos \phi\right)\right),
$$


towards the kinematic endpoint of zero $\Lambda$-velocity in the $\Lambda_{b}$ restframe. As a simple application we may compute the longitudinal lepton polarisation

$$
\left.\left.F_{L}\right|_{\frac{1}{2}}{ }^{+} \rightarrow \frac{1}{2}+\omega_{L}\left(\theta_{\ell}\right)\right\rangle=\frac{\left|H_{0 \frac{1}{2}}\right|^{2}}{\left|H_{0 \frac{1}{2}}\right|^{2}+\left|H_{1 \frac{1}{2}}\right|^{2}}=\frac{\Omega}{3}\left(2 \hat{K}_{1 s s}-\hat{K}_{1 c c}\right) \rightarrow \frac{1}{3},
$$

where $\omega_{L}\left(\theta_{\ell}\right)=2-5 \cos ^{2} \theta_{\ell}$. In $[37,40]$ this observable is denoted $F_{0}$ and $F_{1}=1-F_{0} \rightarrow \frac{2}{3}$ and $F_{H}=\frac{3}{2} F_{1}=\frac{3}{2}\left(1-F_{L}\right) \rightarrow 1$ in the notation of [47]. Other examples are the forward backward asymmetries

$$
\begin{aligned}
& \left.A_{F B}^{\ell}\right|_{\frac{1}{2}^{+} \rightarrow \frac{1}{2}^{+}} \equiv\left\langle\operatorname{sgn} \cos \theta_{\ell}\right\rangle=\frac{\Omega}{2} \hat{K}_{1 c} \rightarrow 0, \\
& \left.A_{F B}^{\Lambda}\right|_{\frac{1}{2}^{+} \rightarrow \frac{1}{2}^{+}}{ }^{+} \equiv\left\langle\operatorname{sgn} \cos \theta_{\Lambda}\right\rangle=\frac{\Omega}{6}\left(\hat{K}_{2 c c}+2 \hat{K}_{2 s s}\right) \rightarrow 0, \\
& \left.A_{F B}^{\Lambda \ell}\right|_{\frac{1}{2}^{+} \rightarrow \frac{1}{2}^{+}}{ }^{+} \equiv\left\langle\operatorname{sgn}\left(\cos \theta_{\ell} \cos \theta_{\Lambda}\right)\right\rangle=\frac{\Omega}{4} \hat{K}_{2 c} \rightarrow-\frac{1}{4} \alpha r .
\end{aligned}
$$

As in $B \rightarrow V \ell^{+} \ell^{+}$the forward backward asymmetries vanish at the endpoint except the combined one which is sensitive to the parity violating decay of the $\Lambda$-baryon. For the $\frac{1}{2}^{+} \rightarrow \frac{1}{2}^{+}$case into dineutrinos, as in $\Lambda_{b} \rightarrow \Lambda(\rightarrow N \pi) \nu \bar{\nu}$, the angular analysis is limited to

$$
\left(\frac{d \Gamma}{d q^{2}}\right)^{-1} \frac{d \Gamma}{d \cos \theta_{\Lambda}}=\hat{K}_{1 c c}+2 \hat{K}_{1 s s}+\left(\hat{K}_{2 c c}+2 \hat{K}_{2 s s}\right) \cos \theta_{\Lambda} \rightarrow 3
$$

which assumes a constant value at the endpoint. Generally one can extract $A_{F B}^{\Lambda}$, which vanishes at the endpoint as predicted in (5.9).

\subsection{The $\Lambda_{J^{P}=\frac{1}{2}}{ }^{+} \rightarrow \Lambda_{J^{P}=\frac{3}{2}}^{\prime}{ }^{ \pm}$angular distribution at the endpoint}

For the $\frac{1}{2}^{+} \rightarrow \frac{3}{2}^{-}$decay the distribution found in [37] is

$$
\begin{aligned}
\hat{L}\left(q^{2}, \theta_{\ell}, \theta_{\Lambda}^{*}, \phi\right)= & \cos ^{2} \theta_{\Lambda}^{*}\left(\hat{L}_{1 c} \cos \theta_{\ell}+\hat{L}_{1 c c} \cos ^{2} \theta_{\ell}+\hat{L}_{1 s s} \sin ^{2} \theta_{\ell}\right) \\
& +\sin ^{2} \theta_{\Lambda}^{*}\left(\hat{L}_{2 c} \cos \theta_{\ell}+\hat{L}_{2 c c} \cos ^{2} \theta_{\ell}+\hat{L}_{2 s s} \sin ^{2} \theta_{\ell}\right) \\
& +\sin ^{2} \theta_{\Lambda}^{*}\left(\hat{L}_{3 s s} \sin ^{2} \theta_{\ell} \cos ^{2} \phi+\hat{L}_{4 s s} \sin ^{2} \theta_{\ell} \sin \phi \cos \phi\right) \\
& +\sin \theta_{\Lambda}^{*} \cos \theta_{\Lambda}^{*} \cos \phi\left(\hat{L}_{5 s} \sin \theta_{\ell}+\hat{L}_{5 s c} \sin \theta_{\ell} \cos \theta_{\ell}\right) \\
& +\sin \theta_{\Lambda}^{*} \cos \theta_{\Lambda}^{*} \sin \phi\left(\hat{L}_{6 s} \sin \theta_{\ell}+\hat{L}_{6 s c} \sin \theta_{\ell} \cos \theta_{\ell}\right),
\end{aligned}
$$

for which $\Lambda_{b} \rightarrow \Lambda(\rightarrow N \bar{K}) \ell^{+} \ell^{-}$was specifically considered. Proceeding in the same way as before using our findings, in section 4 , and inserting them into their expressions for the $L$ 's we get the following

$$
\left\{\hat{L}_{1 c c}, \hat{L}_{1 s s}, \hat{L}_{2 c c}, \hat{L}_{2 s s}, \hat{L}_{3 s s}, \hat{L}_{5 s c}\right\} \rightarrow \frac{1}{\Omega}\left\{\frac{1}{2}, \frac{5}{4}, \frac{5}{4}, \frac{5}{4},-\frac{3}{4},-\frac{3}{2}\right\}
$$

non vanishing contributions which are consistent with the partial list given in eq. 4.14 [37]. Again the one proportional to the imaginary part vanish since all amplitudes are proportional 
to each other. With (5.12) the angular distribution assumes the simple form

$$
\hat{L} \rightarrow \frac{1}{\Omega}\left(\frac{1}{4}\left(5-3 \sin ^{2} \theta_{\Lambda}^{*} \cos ^{2} \phi\right)-\frac{3}{8} \sin 2 \theta_{\ell} \sin 2 \theta_{\Lambda}^{*} \cos \phi-\frac{3 \cos \theta_{\ell}^{2}}{4}\left(\cos ^{2} \theta_{\Lambda}^{*}-\sin ^{2} \theta_{\Lambda}^{*} \cos ^{2} \phi\right)\right),
$$

at the kinematic endpoint of zero $\Lambda^{*}$-velocity in the restframe of the decaying particle. As a simple illustration let us consider the longitudinal polarisation

$$
\begin{aligned}
& \left.F_{L}\right|_{\frac{1}{2}^{+} \rightarrow \frac{3}{2}^{-}} \equiv\left\langle\omega_{L}\left(\theta_{\ell}\right)\right\rangle=\frac{\left|H_{0 \frac{1}{2}}\right|^{2}}{\left|H_{0 \frac{1}{2}}\right|^{2}+\left|H_{1 \frac{1}{2}}\right|^{2}+\left|H_{\overline{1} \frac{3}{2}}\right|^{2}} \\
& =\frac{\Omega}{9}\left(2 \hat{L}_{1 s s}-2 \hat{L}_{2 c c}+4 \hat{L}_{2 s s}+2 \hat{L}_{3 s s}-\hat{L}_{1 c c}\right) \rightarrow \frac{1}{3},
\end{aligned}
$$

for which the same numerical result is obtained as in the $\frac{1}{2}$-case and thus $F_{T}=1-F_{L} \rightarrow \frac{2}{3}$ (and $F_{H}=\frac{3}{2} F_{T} \rightarrow 1$ ). Again the forward backward asymmetry in the lepton angle

$$
\left.A_{F B}\right|_{\frac{1}{2}^{+} \rightarrow \frac{3}{2}^{-}} \equiv\left\langle\operatorname{sgn} \cos \theta_{\ell}\right\rangle=\frac{\Omega}{6}\left(\hat{L}_{1 c}+2 \hat{L}_{2 c}\right) \rightarrow 0,
$$

vanishes. The forward backward asymmetries involving the Baryon angle (cf. (5.9)) vanish, $\left.A_{F B}^{\Lambda}\right|_{\frac{1}{2}^{+} \rightarrow \frac{3}{2}^{-}}=0$ and $\left.A_{F B}^{\Lambda \ell}\right|_{\frac{1}{2}^{+} \rightarrow \frac{3}{2}^{-}}=0$, since the final state Baryon decays via the strong force. The angular analysis for the $\frac{1}{2}^{+} \rightarrow \frac{3}{2}^{-}$case into dineutrinos as in $\Lambda_{b} \rightarrow \Lambda(\rightarrow N \bar{K}) \nu \bar{\nu}$ is restricted to

$$
\left(\frac{d \Gamma}{d q^{2}}\right)^{-1} \frac{d \Gamma}{d \cos \theta_{\Lambda}^{*}}=\left(\hat{L}_{1 c c}+2 \hat{L}_{1 s s}\right) \cos ^{2} \theta_{\Lambda}^{*}+\left(\hat{L}_{2 c c}+2 \hat{L}_{2 s s}+\hat{L}_{3 s s}\right) \sin ^{2} \theta_{\Lambda}^{*} \rightarrow 3
$$

and assumes a constant value at the endpoint.

\subsection{In the vicinity of the endpoint}

Endpoint relations are exact at the kinematic endpoint. However, since experiment provides bins in the $q^{2}$-variable, the question of how endpoint prediction deviate in the vicinity of the endpoint poses itself. We propose to take inspiration from the inclusive $b \rightarrow s \ell^{+} \ell^{-}$ decay. Without QED-correction, the inclusive differential rate, $d \Gamma /\left(d q^{2} d \cos \theta_{\ell}\right)$, consists of three terms: the total rate, $A_{\mathrm{FB}}$ and $F_{L}[50,51]$ out of which $F_{L}$ is the most interesting for our purpose. Since we aim at a rough estimate we restrict ourselves to the tree-level prediction for which

$$
F_{L}(s, \hat{m})=\frac{s-1-\hat{m}^{4}+\hat{m}^{2}(s+2)}{(s-1)(2 s+1)-\hat{m}^{4}-\hat{m}^{2}(s-2)},
$$

$s_{\max }=(1-\hat{m})^{2}, \hat{m}=m_{b} / m_{s}$ for $b \rightarrow s$ and accordingly for other quark level transitions. Note that $F_{L}\left(s_{\max }\right)=1 / 3$ which is just a special case of the $\frac{1}{2} \rightarrow \frac{1}{2}$-transition (5.8). Defining 
$R_{L}(s, \hat{m})=\left(F_{L}(s, \hat{m})-F\left(s_{\max }\right)\right) / F\left(s_{\max }\right)$, one gets the relative deviations

$$
\begin{aligned}
& \left(R_{L}\left(0.8 s_{\max }, \frac{m_{d, u}}{m_{b, c}}\right), R_{L}\left(0.9 s_{\max }, \frac{m_{d, u}}{m_{b, c}}\right)\right) \approx(0.15,0.07), \\
& \left(R_{L}\left(0.8 s_{\max }, \frac{m_{s}}{m_{b}}\right), R_{L}\left(0.9 s_{\max }, \frac{m_{s}}{m_{b}}\right)\right) \quad \approx(0.18,0.09), \\
& \left(R_{L}\left(0.8 s_{\max }, \frac{m_{s}}{m_{c}}\right), R_{L}\left(0.9 s_{\max }, \frac{m_{s}}{m_{c}}\right)\right) \approx(0.22,0.11), \\
& \left(R_{L}\left(0.8 s_{\max }, \frac{m_{c}}{m_{b}}\right), R_{L}\left(0.9 s_{\max }, \frac{m_{c}}{m_{b}}\right)\right) \approx(0.27,0.13),
\end{aligned}
$$

with $m_{d, u} / m_{b, c} \approx 0, m_{s} / m_{b} \approx 0.02, m_{s} / m_{c} \approx 0.08$ and $m_{c} / m_{b} \approx 0.28$ for the $20 \%$ - and $10 \%$-bin towards the endpoint. It is noted that mass effects are sizeable. In what follows we distinguish the semileptonic- and the FCNC-case as the latter are plagued by broad resonances towards the endpoint.

- Semileptonic-case. For $b \rightarrow u, c \rightarrow d$ and $c \rightarrow s$ we may take the first and third line (5.18) at face value and conclude that for a bin at the endpoint, with $(20,10) \%$ of the entire range, the deviation from the endpoint value to be $(8,4) \%$ and $(11,6) \%$ respectively. For $b \rightarrow c$ we see that the effect is roughly doubled in size, as compared to the $u, d$-transitions, with $(16,8) \%$ for the $(20,10) \%$-bin respectively.

- FCNC-case. The $b \rightarrow d, c \rightarrow u$ and $b \rightarrow s$ transitions include in the vicinity of the endpoint broad resonances with potentially different phases. Based on the plot in figure 11 in [52] (see also [53]) we estimate for beauty that the deviation will be $50 \%$ on top of the semileptonic-case. This is $(12,6) \%$ and $(14,7) \%$ for the $(20,10) \%$-range intervals. $^{11}$

These estimates are understood to be valid for baryonic as well as mesonic transitions and therefore fill a gap in our previous work [2] where we did not comment on this aspect. In the semileptonic case of small $\hat{m}$ the corrections are rather small and it is feasible that QED-corrections are competitive. The subject of the latter is though subtle from many viewpoints. For example, the definition of the angular observables will depend on how the photon is treated within the kinematic variables [54] and experiment aims to remove the QED at last in the large logarithms.

\section{$6 \quad$ Summary and conclusions}

We established endpoint relations for baryons based on Lorentz symmetry resulting in exact ratios of helicity amplitudes at the kinematic endpoint cf. eqs. (2.9) and (2.10), for the $\frac{1}{2} \rightarrow \frac{1}{2}$ and $\frac{1}{2} \rightarrow \frac{3}{2}$ baryon transitions respectively. These relations have been verified explicitly, in the form factor basis (3.1) and (3.6), with resuling helicity amplitudes in (3.13) and (3.19)

\footnotetext{
${ }^{11}$ The situation in charm changes drastically in the presence of new physics, which is much less constrained than in beauty. In fact $F_{L}$ tests the Standard Model [25]. A concrete BSM study for $\Lambda_{c} \rightarrow p \mu^{+} \mu^{-}$reveals a possible relative deviation in the $20 \%$ - and $10 \%$-bin of roughly $45 \%$ and $25 \%$, respectively.
} 
respectively. We stress that these relations are valid for long-distance matrix elements such as four-quark operators (leading to resonance contributions). These relations have hitherto been overlooked in the helicity-based form factor analyses since the constraints established in section 4 have not been validated. Exact angular distributions at the kinematic endpoint are derived for the $\frac{1}{2} \rightarrow \frac{1}{2}$ and $\frac{1}{2} \rightarrow \frac{3}{2}$ decays in eqs. (5.6) and (5.12) respectively. These predictions hold model-independently in the $d=6$ weak Hamiltonian. However we wish to emphasise that this framework does not cover light new physics (beyond the Standard Model) or QED-corrections.

We hope that our work contributes to exciting experimental and theoretical effort in baryonic modes which include FCNC and semileptonic modes such as

$$
\begin{aligned}
& \Lambda_{b} \rightarrow \Lambda \ell \ell, \quad \Xi_{b} \rightarrow \Xi \ell \ell, \quad \Lambda_{b} \rightarrow n \ell \ell, \quad \Lambda_{c} \rightarrow p \ell \ell, \\
& \Xi_{c} \rightarrow(\Sigma, \Lambda) \ell \ell, \quad \Omega_{c} \rightarrow \Xi \ell \ell, \quad \Sigma^{+} \rightarrow p \ell \ell, \\
& \Lambda_{b} \rightarrow \Lambda_{c} \ell \nu, \quad \Lambda_{c} \rightarrow \Lambda \ell \nu, \quad \Lambda \rightarrow p \ell \nu, \quad \Xi^{-} \rightarrow\left(\Lambda, \Sigma^{0}\right) \ell \nu,
\end{aligned}
$$

for $\frac{1}{2} \rightarrow \frac{1}{2}$ and $^{12}$

$$
\Lambda_{b} \rightarrow \Lambda^{*} \ell \ell, \quad \Omega_{b} \rightarrow \Omega \ell \ell, \quad \Lambda_{c} \rightarrow N^{+} \ell \ell, \quad \Xi_{c} \rightarrow\left(\Sigma^{*}, \Lambda^{*}\right) \ell \ell, \quad \Omega_{c} \rightarrow \Xi^{*} \ell \ell,
$$

for the $\frac{1}{2} \rightarrow \frac{3}{2}$ case.

\section{Acknowledgments}

We are grateful to Stefan Meinel for asking us the question to what degree endpoint relations hold for $\frac{3}{2}$-baryons, the coordination of his work [13] and for many discussions on the literature. GH is grateful to Marcel Golz and Tom Magorsch for discussions and coordination of their work. RZ is supported by an STFC Consolidated Grant, ST/P0000630/1. The work by GH is supported in part by the Bundesministerium für Bildung und Forschung (BMBF) under project number 05H21PECL2.

\section{A Conventions}

\section{A.1 Polarisation vectors}

We follow our conventions [1-3] for which a complete set of helicity vectors in the restframe is given by ${ }^{13}$

$$
\omega( \pm)=-(0, \pm 1, i, 0) / \sqrt{2}, \quad \omega(0)=(0,0,0,1) \quad \text { and } \quad \omega(t)=(1,0,0,0),
$$

\footnotetext{
${ }^{12}$ We denote with a ' $*$ ' excited hadrons with spin $3 / 2$ and $\mathrm{SU}(3)_{F}$-quantum numbers as the corresponding ground state. A famous example of a $\Lambda^{*}$ is the $\Lambda(1520)$.

${ }^{13}$ The only difference to [1-3] is the sign of $\omega( \pm)$ which we adapt here to match the spinor conventions in appendix A.2. The correctness of the conventions can be established by computing

$$
C_{\lambda_{c} \lambda_{q} \bar{\sigma}}^{\frac{1}{2} 1 \frac{1}{2}} \propto \bar{u}(\sigma) \gamma_{\mu} \gamma_{5} u\left(\lambda_{c}\right) \omega^{* \mu}\left(\lambda_{q}\right)
$$

in the restframe of the spinors (for example). An alternative to the sign change in $\omega( \pm)$ is to introduce a relative minus sign between the two $\lambda= \pm \frac{1}{2}$-spinors.
} 
where indices are understood to by raised. The boosted versions with respect to $q=$ $\left(q_{0}, 0,0, \kappa\right)$ and $p_{p}=\left(E_{p(N)}, 0,0,-\kappa\right)\left(q_{0}=\sqrt{q^{2}+\kappa^{2}}, E_{p(N)}=\sqrt{m_{N}^{2}+\kappa^{2}}\right)$ are

$$
\begin{aligned}
& \beta(q, \pm)=\omega( \pm), \quad \beta(q, 0)=\left(\kappa, 0,0, q_{0}\right) \frac{1}{\sqrt{q^{2}}}, \quad \beta(q, t)=\frac{q}{\sqrt{q^{2}}}, \\
& \eta\left(p_{N}, \pm\right)=\omega(\mp), \quad \eta\left(p_{N}, 0\right)=\left(-\kappa, 0,0, E_{p(N)}\right) \frac{1}{m_{N}}, \quad \eta\left(p_{N}, t\right)=\frac{p_{p}}{m_{N}} .
\end{aligned}
$$

All three sets of helicity vectors satisfy the completeness relation in (2.3). The $\eta$ helicity vector is needed for the $\frac{3}{2}$-spinor as shown further below.

\section{A.2 Dirac algebra}

We choose to work in the Dirac representation of the Clifford algebra

$$
\gamma_{0}=\left(\begin{array}{cc}
1 & 0 \\
0 & -1
\end{array}\right), \quad \gamma_{i}=\left(\begin{array}{cc}
0 & -\sigma^{i} \\
\sigma^{i} & 0
\end{array}\right), \quad \gamma_{5}=\left(\begin{array}{ll}
0 & 1 \\
1 & 0
\end{array}\right),
$$

where $\sigma^{i}$ are the usual $2 \times 2$ Pauli matrices $\gamma_{5}=i \gamma^{0} \gamma^{1} \gamma^{2} \gamma^{3}$ holds, $\sigma_{\mu \nu}=\frac{i}{2}\left[\gamma_{\mu}, \gamma_{\nu}\right]$ and $g_{\mu \nu}=\operatorname{diag}(1,-1,-1,-1)=\frac{1}{2}\left\{\gamma_{\mu}, \gamma_{\nu}\right\}$. With $\varepsilon_{0123}=1$

$$
\sigma^{\alpha \beta} \gamma_{5}=-\frac{i}{2} \varepsilon^{\alpha \beta \gamma \delta} \sigma_{\gamma \delta}
$$

holds. The $\frac{1}{2}$-spinors (Dirac) $u$ and $v$, moving into the $z$-direction, are given by (e.g. [30])

$$
u(\lambda)=\left(\begin{array}{c}
\beta^{+} \chi_{\lambda} \\
2 \lambda \beta^{-} \chi_{\lambda}
\end{array}\right), \quad v(\lambda)=\left(\begin{array}{c}
\beta^{-} \chi_{\lambda} \\
-2 \lambda \beta^{+} \chi_{\lambda}
\end{array}\right)=C \bar{u}^{T}
$$

with $\chi_{\frac{1}{2}}=(1,0), \chi_{-\frac{1}{2}}=(0,1)$ and $\beta^{ \pm} \equiv \sqrt{E \pm m}$ (with $\hat{s}_{ \pm}=2\left(\hat{\beta}^{ \pm}\right)^{2}$ in the notation of this paper). If the direction of movement is reversed then $\beta^{-} \rightarrow-\beta^{-}$in the formula above. For generic directions see the reference above. In (A.5) $C=i \gamma^{0} \gamma^{2}$ is the charge conjugation matrix in the Dirac representation.

\section{A.3 The $\frac{3}{2}$-spinor}

The spin 3/2-states were first discussed by Rarita and Schwinger [55]. It can be constructed out of the spin one polarisation tensor and the Dirac spinor using the Clebsch-Gordan coeffcients

$$
\Psi_{\alpha}\left(p_{N}, \lambda\right)=\sum_{\sigma=-1 / 2}^{1 / 2} C_{\lambda s(\lambda-\sigma)}^{\frac{3}{2} \frac{1}{2} 1} \eta_{\alpha}\left(p_{N}, \lambda-\sigma\right) u\left(p_{N}, \sigma\right),
$$

and $p_{N}^{\alpha} \Psi_{\alpha}=0$ holds trivially and $\gamma^{\alpha} \Psi_{\alpha}=0$ can be verified using the conventions used in the previous sections. The normalisation is such that $\bar{\Psi}^{\alpha}\left(p_{N}, \lambda\right) \Psi_{\alpha}\left(p_{N}, \lambda^{\prime}\right)=-2 m_{N} \delta_{\lambda, \lambda^{\prime}}$. Again the CGC are the ones compatible with the Condon-Shortley phase convention and can be looked up in the [33]. 


\section{B The tensor form factor relation due to $\sigma^{\alpha \beta} \gamma_{5}=-\frac{i}{2} \varepsilon^{\alpha \beta \gamma \delta} \sigma_{\gamma \delta}$}

In the form factor definitions (3.1) and (3.6) we have contracted the $\sigma_{\mu \nu}$ with $q^{\nu}$, as is commonly done in the literature. This leads to relations at $q^{2}=0$ since the form factor without contraction is free from poles in $q^{2}$. In some sense these relations, which we derive below, can be seen as an artefact of the parametrisation. Further note that at $q^{2}=0$ the zero-helicity part decouples as one would expect from a massless particle. The constraints are thus necessarily between form factors in the perpendicular or transversal directions.

\section{B.1 $\Lambda_{J^{P}=\frac{1}{2}}+\rightarrow \Lambda_{J^{P}=\frac{1}{2}}^{\prime}{ }^{- \text {-case }}$}

Here we aim to demonstrate $t_{2}^{V}(0)=t_{2}^{A}(0)$ of the form factors defined in (3.1). The most general form of the uncontracted version of the tensor form factors reads

$$
\begin{aligned}
& \left\langle p\left(p_{p}, \sigma\right)\left|\bar{s} i \sigma^{\mu \nu}\left(\gamma_{5}\right) b\right| \Lambda_{c}\left(p, \lambda_{c}\right)\right\rangle= \\
& \quad \bar{u}\left(p_{p}, \sigma\right)\left(x_{A}\left(q^{2}\right) \hat{p}_{[\mu} \hat{q}_{\nu]}-x_{B}\left(q^{2}\right) i \sigma_{\mu \nu}+x_{C}\left(q^{2}\right) \gamma_{[\mu} \hat{p}_{\nu]}+x_{D}\left(q^{2}\right) \gamma_{[\mu} \hat{q}_{\nu]}\right) u\left(p, \lambda_{c}\right),
\end{aligned}
$$

where the square brackets denote antisymmetrisation as in $a_{[\alpha} b_{\beta]}=a_{\alpha} b_{\beta}-a_{\beta} b_{\alpha}$. Note that due to the relation

$$
\left\{\sigma_{\alpha \beta}, \sigma_{\mu \nu}\right\}=2\left(\left(g_{\alpha \mu} g_{\beta \nu}-\{\alpha \leftrightarrow \beta\}\right)-i \epsilon_{\alpha \beta \mu \nu} \gamma_{5}\right)
$$

which can be derived from the Chisholm identity for example, the $\epsilon_{\mu \nu p q} \bar{u}\left(p_{p}, \sigma\right) \gamma_{5} u\left(p, \lambda_{c}\right)$ structure can be expressed in terms of the others. Using the identity (A.4) and the definitions (3.1) ones arrives at the following identifications

$$
\begin{array}{ll}
t_{1}^{V}\left(q^{2}\right)=x_{D}\left(q^{2}\right)+\frac{1}{2}\left(x_{C}\left(q^{2}\right)+\hat{\Delta}_{+} x_{A}\left(q^{2}\right)\right), & t_{1}^{A}\left(q^{2}\right)=\frac{1}{2} x_{C}\left(q^{2}\right) \\
t_{2}^{V}\left(q^{2}\right)=x_{B}\left(q^{2}\right)+\frac{1}{2}\left(\hat{\Delta}_{-} x_{C}\left(q^{2}\right)-2 \hat{q}^{2} x_{A}\left(q^{2}\right)\right), & t_{2}^{A}\left(q^{2}\right)=x_{B}\left(q^{2}\right)+\frac{1}{2} \hat{\Delta}_{-} x_{C}\left(q^{2}\right) .
\end{array}
$$

Since all $x_{i}$, and in particular $x_{A}$ are non-singular for $q^{2} \rightarrow 0$ it follows that

$$
t_{2}^{V}(0)=t_{2}^{A}(0)
$$

which we aimed to show. This relation has been obtained previously in [32] albeit in the specific context of their computation.

\section{B.2 $\Lambda_{J^{P}=\frac{1}{2}}+\Lambda_{J^{P}=\frac{3}{2}}^{\prime}{ }^{- \text {-case }}$}

Proceeding in complete analogy we write the most generic ansatz for (3.6) with uncontracted $q$

$$
\begin{aligned}
\left\langle N\left(p^{\prime}, \sigma\right)\left|\bar{s} i \sigma^{\mu \nu} b\right| \Lambda_{c}\left(p, \lambda_{c}\right)\right\rangle= & \bar{\Psi}_{\alpha}\left(p^{\prime}, \sigma\right) i\left\{\hat{p}_{\alpha}\left(X_{A}\left(-i \sigma_{\mu \nu}\right)+X_{B} \gamma_{[\mu} \hat{p}_{\nu]}+X_{C} \gamma_{[\mu} \hat{p}_{\nu]}^{\prime}+X_{D} \hat{p}_{[\mu} \hat{p}_{\nu]}^{\prime}\right)\right. \\
& \left.+X_{E} g_{\alpha[\mu} \gamma_{\nu]}+X_{F} g_{\alpha[\mu} p_{\nu]}+X_{G} g_{\alpha[\mu} p_{\nu]}^{\prime}\right\} u\left(p, \lambda_{c}\right),
\end{aligned}
$$


where here $p^{\prime}=p_{N}$ and $X_{i}=X_{i}\left(q^{2}\right)$ for brevity and we have chosen the $\frac{1}{2}^{-} \rightarrow \frac{3}{2}^{-}$-transition for concreteness. In reducing the structures we made use of (B.2) and the equation of motion. Contracting with $q$ and equating with our ansatz (3.6) ones finds

$$
\begin{aligned}
& T_{1}^{V}=2 X_{A}+\hat{\Delta}_{-}\left(X_{B}+X_{C}\right)+q^{2} X_{D}+X_{F}+X_{G}, \\
& T_{2}^{V}=-\hat{\Delta}_{+} X_{A}+\frac{1}{2}\left(\hat{m}_{N}^{2}-\hat{q}^{2}-1\right) X_{B}+\frac{1}{2}\left(\hat{m}_{N}^{2}+\hat{q}^{2}-1\right) X_{C}+X_{E}, \\
& T_{3}^{V}=-X_{A}+\hat{\Delta}_{-} X_{B}-\frac{1}{2}\left(\hat{m}_{N}^{2}+\hat{q}^{2}-1\right) X_{D}-X_{F} \\
& T_{1}^{A}=2 X_{A}+\hat{\Delta}_{-}\left(X_{B}+X_{C}\right)+X_{F}+X_{G}, \\
& T_{2}^{A}=-\hat{\Delta}_{-} X_{A}+\frac{1}{2}\left(\hat{q}^{2}+2 \hat{m}_{N}-\hat{m}_{N}^{2}-1\right)\left(X_{B}+X_{C}\right)+X_{E}+\hat{m}_{N}\left(X_{F}+X_{G}\right), \\
& T_{3}^{A}=-X_{A}+\hat{m}_{N}\left(X_{B}+X_{C}\right) .
\end{aligned}
$$

At $q^{2}=0$ the following constraints emerge

$$
\frac{1}{2}^{+} \rightarrow \frac{3}{2}^{\mp}: \quad T_{1}^{V}(0)=T_{1}^{A}(0), \quad T_{2}^{A(V)}(0)=T_{2}^{V(A)}(0)+\hat{m}_{N} T_{1}^{V(A)}(0),
$$

in accordance with $(3.9)$ and $[37,39]$. The new aspect is that the relation (B.7) has been derived with seven structures as advocated in [38]. In [37] only six structures have been considered and nn [39] no detail has been given.

Open Access. This article is distributed under the terms of the Creative Commons Attribution License (CC-BY 4.0), which permits any use, distribution and reproduction in any medium, provided the original author(s) and source are credited.

\section{References}

[1] R. Zwicky, Endpoint symmetries of helicity amplitudes, arXiv:1309.7802 [INSPIRE].

[2] G. Hiller and R. Zwicky, (A)symmetries of weak decays at and near the kinematic endpoint, JHEP 03 (2014) 042 [arXiv:1312.1923] [InSPIRE].

[3] J. Gratrex, M. Hopfer and R. Zwicky, Generalised helicity formalism, higher moments and the $B \rightarrow K_{J_{K}}(\rightarrow K \pi) \bar{\ell}_{1} \ell_{2}$ angular distributions, Phys. Rev. D 93 (2016) 054008 [arXiv: 1506.03970] [INSPIRE].

[4] G. Buchalla et al., B, D and $K$ decays, Eur. Phys. J. C 57 (2008) 309 [arXiv:0801.1833] [INSPIRE].

[5] HFLAV collaboration, Averages of b-hadron, c-hadron, and $\tau$-lepton properties as of 2018 , Eur. Phys. J. C 81 (2021) 226 [arXiv:1909.12524] [INSPIRE].

[6] G. Hiller, A Challenge to Lepton Universality, APS Physics 7 (2014) 102.

[7] S. Bifani, S. Descotes-Genon, A. Romero Vidal and M.-H. Schune, Review of Lepton Universality tests in B decays, J. Phys. G 46 (2019) 023001 [arXiv:1809.06229] [INSPIRE].

[8] LHCb collaboration, Test of lepton universality with $\Lambda_{b}^{0} \rightarrow p K^{-} \ell^{+} \ell^{-}$decays, JHEP 05 (2020) 040 [arXiv: 1912.08139] [INSPIRE]. 
[9] LHCb collaboration, Test of lepton universality in beauty-quark decays, arXiv:2103.11769 [INSPIRE].

[10] W. Detmold and S. Meinel, $\Lambda_{b} \rightarrow \Lambda \ell^{+} \ell^{-}$form factors, differential branching fraction, and angular observables from lattice QCD with relativistic b quarks, Phys. Rev. D 93 (2016) 074501 [arXiv: 1602.01399] [INSPIRE].

[11] S. Meinel, $\Lambda_{c} \rightarrow \Lambda l^{+} \nu_{l}$ form factors and decay rates from lattice QCD with physical quark masses, Phys. Rev. Lett. 118 (2017) 082001 [arXiv:1611.09696] [INSPIRE].

[12] Q.-A. Zhang et al., $\Xi_{c} \rightarrow \Xi$ Form Factors and $\Xi_{c} \rightarrow \Xi \ell^{+} \nu_{\ell}$ Decay Rates From Lattice QCD, arXiv:2103.07064 [INSPIRE].

[13] S. Meinel and G. Rendon, $\Lambda_{c} \rightarrow \Lambda^{*}(1520)$ form factors from lattice $Q C D$ and improved analysis of the $\Lambda_{b} \rightarrow \Lambda^{*}(1520)$ and $\Lambda_{b} \rightarrow \Lambda_{c}^{*}(2595,2625)$ form factors, arXiv:2107.13140 [INSPIRE].

[14] H. Bahtiyar, $\Lambda_{c} \rightarrow \Lambda$ Form Factors in Lattice QCD, Turk. J. Phys. 45 (2021) 4 [arXiv:2107.13909] [INSPIRE].

[15] K. Azizi, Y. Sarac and H. Sundu, Light cone QCD sum rules study of the semileptonic heavy $\Xi_{Q}$ and $\Xi_{Q}^{\prime}$ transitions to $\Xi$ and $\Sigma$ baryons, Eur. Phys. J. A 48 (2012) 2 [arXiv:1107.5925] [INSPIRE].

[16] Y.-M. Wang and Y.-L. Shen, Perturbative Corrections to $\Lambda_{b} \rightarrow \Lambda$ Form Factors from QCD Light-Cone Sum Rules, JHEP 02 (2016) 179 [arXiv:1511.09036] [INSPIRE].

[17] A. Cerri et al., Report from Working Group 4: Opportunities in Flavour Physics at the HL-LHC and HE-LHC, CERN Yellow Rep. Monogr. 7 (2019) 867 [arXiv:1812.07638] [INSPIRE].

[18] BeLle-II collaboration, The Belle II Physics Book, PTEP 2019 (2019) 123 C01 [Erratum ibid. 2020 (2020) 029201] [arXiv: 1808.10567] [INSPIRE].

[19] BESIII collaboration, Future Physics Programme of BESIII, Chin. Phys. C 44 (2020) 040001 [arXiv: 1912.05983] [INSPIRE].

[20] FCC collaboration, FCC Physics Opportunities: Future Circular Collider Conceptual Design Report Volume 1, Eur. Phys. J. C 79 (2019) 474 [inSPIRE].

[21] A.A. Alves Junior et al., Prospects for Measurements with Strange Hadrons at LHCb, JHEP 05 (2019) 048 [arXiv: 1808.03477] [INSPIRE].

[22] BABAR collaboration, Searches for Rare or Forbidden Semileptonic Charm Decays, Phys. Rev. D 84 (2011) 072006 [arXiv: 1107.4465] [INSPIRE].

[23] LHCb collaboration, Search for the rare decay $\Lambda_{c}^{+} \rightarrow p \mu^{+} \mu^{-}$, Phys. Rev. D 97 (2018) 091101 [arXiv: 1712.07938] [INSPIRE].

[24] S. Meinel, $\Lambda_{c} \rightarrow N$ form factors from lattice $Q C D$ and phenomenology of $\Lambda_{c} \rightarrow n \ell^{+} \nu_{\ell}$ and $\Lambda_{c} \rightarrow p \mu^{+} \mu^{-}$decays, Phys. Rev. D 97 (2018) 034511 [arXiv:1712.05783] [InSPIRE].

[25] M. Golz, G. Hiller and T. Magorsch, Probing for New Physics with Rare Charm Baryon $\left(\Lambda_{c}\right.$, $\left.\Xi_{c}, \Omega_{c}\right)$ Decays, JHEP 09 (2021) 208 [arXiv:2107.13010] [INSPIRE].

[26] LHCb collaboration, Angular moments of the decay $\Lambda_{b}^{0} \rightarrow \Lambda \mu^{+} \mu^{-}$at low hadronic recoil, JHEP 09 (2018) 146 [arXiv: 1808.00264] [INSPIRE]. 
[27] Y. Amhis, S. Descotes-Genon, C. Marin Benito, M. Novoa-Brunet and M.-H. Schune, Prospects for New Physics searches with $\Lambda_{\mathrm{b}}^{0} \rightarrow \Lambda(1520) \ell^{+} \ell^{-}$decays, Eur. Phys. J. Plus 136 (2021) 614 [arXiv: 2005.09602] [INSPIRE].

[28] F. Legger and T. Schietinger, Photon helicity in $\Lambda_{b} \rightarrow p K \gamma$ decays, Phys. Lett. B 645 (2007) 204 [Erratum ibid. 647 (2007) 527] [hep-ph/0605245] [INSPIRE].

[29] M. Jacob and G.C. Wick, On the General Theory of Collisions for Particles with Spin, Annals Phys. 7 (1959) 404 [INSPIRE].

[30] H.E. Haber, Spin formalism and applications to new physics searches, in 21st Annual SLAC Summer Institute on Particle Physics: Spin Structure in High-energy Processes (School: 26 Jul-3 Aug, Topical Conference: 4-6 Aug) (SSI 93), pp. 231-272 (1994) [hep-ph/9405376] [INSPIRE].

[31] A. Faessler, T. Gutsche, M.A. Ivanov, J.G. Korner and V.E. Lyubovitskij, The Exclusive rare decays $B \rightarrow K\left(K^{*}\right) \bar{\ell} \ell$ and $B_{c} \rightarrow D\left(D^{*}\right) \bar{\ell} \ell$ in a relativistic quark model, Eur. Phys. J. direct 4 (2002) 18 [hep-ph/0205287] [INSPIRE].

[32] T. Gutsche, M.A. Ivanov, J.G. Korner, V.E. Lyubovitskij and P. Santorelli, Rare baryon decays $\Lambda_{b} \rightarrow \Lambda l^{+} l^{-}(l=e, \mu, \tau)$ and $\Lambda_{b} \rightarrow \Lambda \gamma$ : differential and total rates, lepton- and hadron-side forward-backward asymmetries, Phys. Rev. D 87 (2013) 074031 [arXiv:1301.3737] [INSPIRE].

[33] Particle Data Group collaboration, Review of Particle Physics, PTEP 2020 (2020) 083C01 [INSPIRE].

[34] M. Wirbel, B. Stech and M. Bauer, Exclusive Semileptonic Decays of Heavy Mesons, Z. Phys. C 29 (1985) 637 [INSPIRE].

[35] A. Bharucha, D.M. Straub and R. Zwicky, $B \rightarrow V \ell^{+} \ell^{-}$in the Standard Model from light-cone sum rules, JHEP 08 (2016) 098 [arXiv: 1503. 05534] [INSPIRE].

[36] T. Gutsche, M.A. Ivanov, J.G. Körner, V.E. Lyubovitskij, V.V. Lyubushkin and P. Santorelli, Theoretical description of the decays $\Lambda_{b} \rightarrow \Lambda^{(*)}\left(\frac{1}{2}^{ \pm}, \frac{3}{2}^{ \pm}\right)+J / \psi$, Phys. Rev. D 96 (2017) 013003 [arXiv: 1705.07299] [INSPIRE].

[37] S. Descotes-Genon and M. Novoa-Brunet, Angular analysis of the rare decay $\Lambda_{b} \rightarrow \Lambda(1520)(\rightarrow N K) \ell^{+} \ell^{-}$, JHEP 06 (2019) 136 [Erratum ibid. 06 (2020) 102] [arXiv: 1903.00448] [INSPIRE].

[38] M. Papucci and D.J. Robinson, Form Factor Counting and HQET Matching for New Physics in $\Lambda_{b} \rightarrow \Lambda_{c}^{*} l \nu$, arXiv:2105.09330 [INSPIRE].

[39] G. Hiller, M. Knecht, F. Legger and T. Schietinger, Photon polarization from helicity suppression in radiative decays of polarized $\Lambda_{b}$ to spin-3/2 baryons, Phys. Lett. B 649 (2007) 152 [hep-ph/0702191] [INSPIRE].

[40] P. Böer, T. Feldmann and D. van Dyk, Angular Analysis of the Decay $\Lambda_{b} \rightarrow \Lambda(\rightarrow N \pi) \ell^{+} \ell^{-}$, JHEP 01 (2015) 155 [arXiv:1410.2115] [InSPIRE].

[41] T. Feldmann and M.W.Y. Yip, Form factors for $\Lambda_{b} \rightarrow \Lambda$ transitions in the soft-collinear effective theory, Phys. Rev. D 85 (2012) 014035 [Erratum ibid. 86 (2012) 079901] [arXiv: 1111.1844] [INSPIRE].

[42] S. Meinel and G. Rendon, $\Lambda_{b} \rightarrow \Lambda^{*}(1520) \ell^{+} \ell^{-}$form factors from lattice $Q C D$, Phys. Rev. $D$ 103 (2021) 074505 [arXiv:2009.09313] [INSPIRE]. 
[43] S. Weinberg, The Quantum theory of fields. Vol. 1: Foundations, Cambridge University Press (2005).

[44] R.J. Eden, P.V. Landshoff, D.I. Olive and J.C. Polkinghorne, The analytic S-matrix, Cambridge University Press, Cambridge (1966).

[45] R. Zwicky, A brief Introduction to Dispersion Relations and Analyticity, in Quantum Field Theory at the Limits: from Strong Fields to Heavy Quarks, pp. 93-120 (2017) [DOI] [arXiv: 1610.06090] [INSPIRE].

[46] W. Detmold, C. Lehner and S. Meinel, $\Lambda_{b} \rightarrow p \ell^{-} \bar{\nu}_{\ell}$ and $\Lambda_{b} \rightarrow \Lambda_{c} \ell^{-} \bar{\nu}_{\ell}$ form factors from lattice QCD with relativistic heavy quarks, Phys. Rev. D 92 (2015) 034503 [arXiv: 1503.01421] [INSPIRE].

[47] P. Böer, M. Bordone, E. Graverini, P. Owen, M. Rotondo and D. Van Dyk, Testing lepton flavour universality in semileptonic $\Lambda_{b} \rightarrow \Lambda_{c}^{*}$ decays, JHEP 06 (2018) 155 [arXiv: 1801.08367] [INSPIRE].

[48] M. Bordone, Heavy Quark Expansion of $\Lambda_{b} \rightarrow \Lambda^{*}$ (1520) Form Factors beyond Leading Order, Symmetry 13 (2021) 531 [arXiv:2101.12028] [INSPIRE].

[49] T. Blake and M. Kreps, Angular distribution of polarised $\Lambda_{b}$ baryons decaying to $\Lambda \ell^{+} \ell^{-}$, JHEP 11 (2017) 138 [arXiv:1710.00746] [INSPIRE].

[50] S. Fukae, C.S. Kim, T. Morozumi and T. Yoshikawa, A Model independent analysis of the rare $B$ decay $B \rightarrow X_{s} l^{+} l^{-}$, Phys. Rev. D 59 (1999) 074013 [hep-ph/9807254] [INSPIRE].

[51] K.S.M. Lee, Z. Ligeti, I.W. Stewart and F.J. Tackmann, Extracting short distance information from $b \rightarrow s l^{+} l^{-}$effectively, Phys. Rev. D 75 (2007) 034016 [hep-ph/0612156] [INSPIRE].

[52] J. Lyon and R. Zwicky, Resonances gone topsy turvy - the charm of QCD or new physics in $b \rightarrow s \ell^{+} \ell^{-}$?, arXiv:1406.0566 [INSPIRE].

[53] S. Braß, G. Hiller and I. Nisandzic, Zooming in on $B \rightarrow K^{*} \ell \ell$ decays at low recoil, Eur. Phys. J. $C \mathbf{7 7}$ (2017) 16 [arXiv:1606.00775] [InSPIRE].

[54] G. Isidori, S. Nabeebaccus and R. Zwicky, QED corrections in $\bar{B} \rightarrow \bar{K} \ell^{+} \ell^{-}$at the double-differential level, JHEP 12 (2020) 104 [arXiv:2009.00929] [INSPIRE].

[55] W. Rarita and J. Schwinger, On a theory of particles with half integral spin, Phys. Rev. 60 (1941) 61 [INSPIRE]. 